\title{
Sema4C/PlexinB2 signaling controls breast cancer cell growth, hormonal dependence and tumorigenic potential
}

\author{
Sreeharsha Gurrapu' ${ }^{1}$ Emanuela Pupo ${ }^{2} \cdot$ Giulia Franzolin $^{1,3} \cdot$ Letizia Lanzetti ${ }^{2,3} \cdot$ Luca Tamagnone $\mathbb{C}^{1,3}$
}

Received: 23 November 2017 / Revised: 21 February 2018 / Accepted: 27 February 2018 / Published online: 19 March 2018

(c) ADMC Associazione Differenziamento e Morte Cellulare 2018

\begin{abstract}
Semaphorin 4C (Sema4C) expression in human breast cancers correlates with poor disease outcome. Surprisingly, upon knock-down of Sema4C or its receptor PlexinB2 in diverse mammary carcinoma cells (but not their normal counterparts), we observed dramatic growth inhibition associated with impairment of G2/M phase transition, cytokinesis defects and the onset of cell senescence. Mechanistically, we demonstrated a Sema4C/PlexinB2/LARG-dependent signaling cascade that is required to maintain critical RhoA-GTP levels in cancer cells. Interestingly, we also found that Sema4C upregulation in luminal-type breast cancer cells drives a dramatic phenotypic change, with disassembly of polarity complexes, mitotic spindle misorientation, cell-cell dissociation and increased migration and invasiveness. We found that this signaling cascade is dependent on the PlexinB2 effectors ErbB2 and RhoA-dependent kinases. Moreover, Sema4C-overexpressing luminal breast cancer cells upregulated the transcription factors Snail, Slug and SOX-2, and formed estrogen-independent metastatic tumors in mice. In sum, our data indicate that Sema4C/PlexinB2 signaling is essential for the growth of breast carcinoma cells, featuring a novel potential therapeutic target. In addition, elevated Sema4C expression enables indolent luminal-type tumors to become resistant to estrogen deprivation, invasive and metastatic in vivo, which could account for its association with a subset of human breast cancers with poor prognosis.
\end{abstract}

\section{Introduction}

Semaphorins constitute a large family of membrane-bound and secreted proteins initially discovered as repelling cues for axons, but then found to regulate a range of biological processes from development to tumor progression [1,2]. Semaphorins can elicit a so-called "forward" signaling cascade through the intracellular domain of receptors in the

Edited by S. Fulda.

Electronic supplementary material The online version of this article (https://doi.org/10.1038/s41418-018-0097-4) contains supplementary material, which is available to authorized users.

Luca Tamagnone

luca.tamagnone@ircc.it

1 Cancer Cell Biology Laboratory, Candiolo Cancer Institute-FPO, IRCCS, Candiolo, Italy

2 Membrane Trafficking Laboratory, Candiolo Cancer InstituteFPO, IRCCS, Candiolo, Italy

3 Department of Oncology, University of Torino Medical School, Candiolo, Turin, Italy
Plexin family, controlling integrin-mediated adhesion and cytoskeletal remodeling [3]. Moreover, transmembrane semaphorins can mediate "reverse" signaling cascades mediated by their own cytoplasmic domains [4].

Cancer cells express both semaphorins and their receptors, and these signals control several hallmarks of cancer, such as cell proliferation and survival, tumor angiogenesis, and evasion from the immune response [2, 5, 6]. Moreover, the expression of semaphorins and their receptors is often upregulated or downregulated in tumors compared with normal tissues. Here we focused our attention on transmembrane Semaphorin 4C (Sema4C), for which aberrant expression has been reported in esophageal, gastric and colorectal cancers [7]. Previous studies have associated Sema4C signaling with the induction of epithelial-mesenchymal transition (EMT), as well as cancer cell resistance to cisplatin and paclitaxel, but the implicated mechanisms remained unclear [8-10]. In addition to tumor cells, Sema4C expression has been found in lymphatic endothelial cells, whereby it promoted lymphatic metastases [11]. PlexinB2 has been recognized as the specific receptor of Sema4C [12, 13]. Previous studies have established PlexinB2 as a major regulator of neural progenitor cells [12, 
14] and developing epithelia [15, 16], but its role in cancer cells awaits elucidation. Interestingly, B-family plexins were found to recruit and control guanine nucleotide exchanger factors (GEFs) for the small GTPase RhoA, such as p190-PRG and LARG [17-19]. Notably, RhoA is highly expressed in human tumors compared with adjacent normal tissues $[20,21]$ and several studies suggest its relevance in tumor growth, invasion and metastasis [22-26]; however, the specificity of this mechanism needs to be elucidated.

Our study provides novel evidence for understanding the functional relevance of Sema4C and its receptor PlexinB2 in cancer. In particular, by gene knock-down experiments we demonstrated an essential role of Sema4C/ PlexinB2 signaling for proliferation and cytokinesis in diverse breast carcinoma cells. We showed that this function depends on a forward signaling cascade mediated by PlexinB2 intracellular domain, featuring a novel kind of "addiction" to critical RhoA-GTP levels in cancer cells. Sema4C overexpression in MCF-7 luminal-type breast cancer cells induced ErbB2- and RhoA-dependent disassembly of cell polarity complexes and mitotic spindle misorientation, facilitating cell detachment from the monolayer and mammosphere formation. Sema4Coverexpressing luminal breast cancer cells furthermore attained estrogen-independent growth and formed metastatic tumors in mice. These properties could account for the observed correlation between particularly elevated Sema4C expression and poor outcome in breast cancer.

\section{Results}

\section{Sema4C is widely expressed in human breast cancers and its elevated expression is associated with poor patient prognosis}

Breast cancer is the most frequent tumor in women. Despite remarkable success in its treatment, the patients are still plagued by a residual risk of poor outcome that cannot be reliably predicted based on current knowledge. We noticed that, in a previously reported 70 -gene classifier predicting breast cancer patient survival, Sema4C levels were significantly higher in the subgroup characterized by poor outcome [27]. Notably, both Sema4C and its receptor PlexinB2 are widely expressed in human breast cancers, although at variable levels, and are commonly upregulated compared with adjacent normal tissues (Suppl. Figure 1A, $\mathrm{B}, \mathrm{C})$. Indeed, by analyzing gene expression in a panel of 1402 clinically annotated breast cancer samples, we found a significant overall association between higher Sema4C levels and poor prognosis (Fig. 1a). Consistent results were furthermore obtained analyzing The Cancer Genome Atlas (TCGA) breast cancer dataset, which includes a tumor subgroup with elevated Sema4C and shorter overall survival ( 84 vs. 130 months; $p=0.016$ ). The correlation between PlexinB2 levels and poor breast cancer prognosis in these datasets did not reach statistical significance, although a positive trend could be seen (Suppl. Figure 1D).

Interestingly, Fig. 1b shows that Sema4C and PlexinB2 are widely expressed in breast cancer cells of different subtypes [28]; in contrast, both proteins were barely detectable in immortalized normal mammary epithelial cells (HMEC-hTERT). Notably, transmembrane semaphorins can undergo proteolytic cleavage on the cell surface, and we found Sema4C shed in the conditioned medium of tumor cells (Fig. 1c). Thus, in breast cancer cells Sema4C exists as secreted and transmembrane isoforms, and it could possibly act by both "forward" and "reverse" signaling mechanisms.

\section{Sema4C/PlexinB2 signaling blockade in breast cancer cells causes cytokinesis defects, growth arrest and cell senescence}

In order to assess the functional role of Sema4C and PlexinB2, we performed gene knock-down in breast cancer cells of different subtype: MCF-7 (luminal, estrogen dependent), SKBR-3 (with constitutive activation of HER2 oncogene) and MDA-MB-231 (triple-negative basal like). In addition, we forcedly expressed a truncated version of PlexinB2 lacking the intracellular domain (PlexinB2-DN), which could act as dominant-negative interfering with endogenous receptor signaling. Quantitative reverse transcriptase-PCR (qRT-PCR) and immunoblotting analysis validated the efficacy of two distinct short hairpin RNA (shRNA) sequences targeting each gene under study (Suppl. Figure 2A, B), and the expression of PlexinB2-DN construct (Supp. Figure 2C). Surprisingly, although cells carrying control shRNAs behaved normally, either Sema4C or PlexinB2 knock-down (or the overexpression of PlexinB2$\mathrm{DN})$ led to a dramatic phenotype: the vast majority of the cells stopped proliferating (Figs. 2a-c; and Suppl. Figure $2 \mathrm{~A}, \mathrm{~B}, \mathrm{D}$ ), although they remained alive (assessed by Trypan blue staining, data not shown), spread and well attached to the substrate. Thus, both Sema4C and PlexinB2 are required for the growth of breast cancer cells of different subtypes, including those carrying a constitutively active oncogene. Importantly, Sema4C or PlexinB2 silencing did not affect the proliferation of non-tumoral cells, such as HMEC-hTERT (Fig. 2d). Of note, the superimposable effect mediated by the dominant-negative PlexinB2 receptor suggested the involvement of a forward semaphorin signaling cascade, elicited by Sema4C ectodomain via the intracellular portion of the plexin. To validate this hypothesis, we assayed functional rescue experiments by providing a recombinant soluble Sema4C ectodomain to cells deprived of endogenous Sema4C (Fig. 2e) or PlexinB2 
A

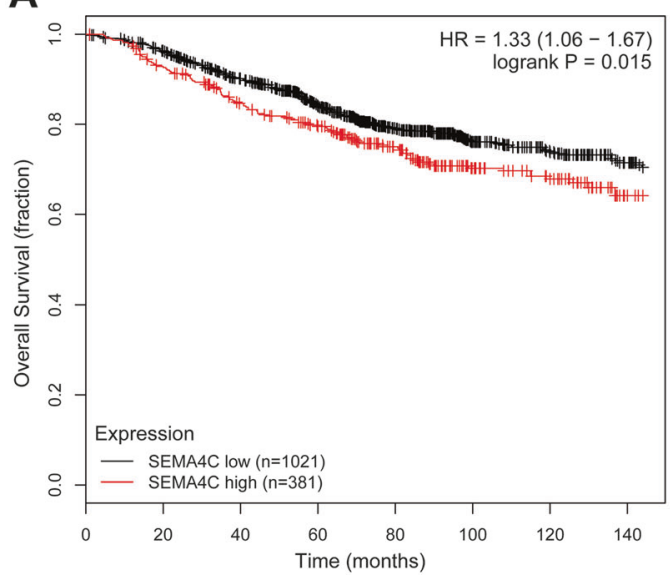

Fig. 1 Sema4C is widely expressed in human breast cancers and its elevated expression is associated with poor patient prognosis. a Kaplan-Meier analysis of overall patient survival (OS; follow-up at 12 years) of unselected breast cancer patients $(n=1402)$ correlation to Semaphorin 4C (Sema4C) high and low expression subgroups, based on online dataset analysis by http://kmplot.com; $p=0.015$. b Western

(Fig. 2f); this was sufficient to rescue the viability of Sema4C-depleted cells, but could not protect cells deprived of PlexinB2 receptor.

In order to elucidate the mechanisms responsible for the observed proliferation defects, we analyzed cell behavior by live microscopy at an early stage after gene targeting (Suppl. Figure 3A and Supplemental Movies 1, 2, 3, 4). Although control cells steadily grew and almost reached confluency in $72 \mathrm{~h}$, cells subjected to Sema4C/PlexinB2 interference rarely entered cell division, and eventually adopted a flat morphology (Figs. 3a, b and Suppl. Figure $4 \mathrm{~A}, \mathrm{~B}$ ); in particular, MDA-MB-231 cells attained a covered area up to 6-8 times larger than controls (Fig. 3c). The observed cell size enlargement and flat morphology was associated with a significant increase in the senescenceassociated marker $\beta$-galatosidase (SA- $\beta$-gal) compared with control cells (Fig. 3d). Moreover, DAPI staining revealed a large fraction of multinucleated cells (Figs. 3e, f). We thus postulated a failure to complete cell division upon Sema4C or PlexinB2 knock-down. In order to address this hypothesis, we analyzed the cell cycle by cytofluorimetry soon after cell transduction, before the onset of cell senescence. This revealed a markedly decreased fraction of cells in G1 phase (consistent with proliferation arrest), associated with increased G2-phase cell fraction, indicating a G2/M transition block (Fig. 3g). Sema4C or PlexinB2 knock-down also resulted in reduced expression of two important regulators of cell cycle and G2/M transition, CCNA2 and CDC2 (CDK1) [29, 30] (Fig. 3h). These data are consistent with a blockade of cytokinesis leading to the accumulation of multiple nuclei in enlarged cell bodies, and to the
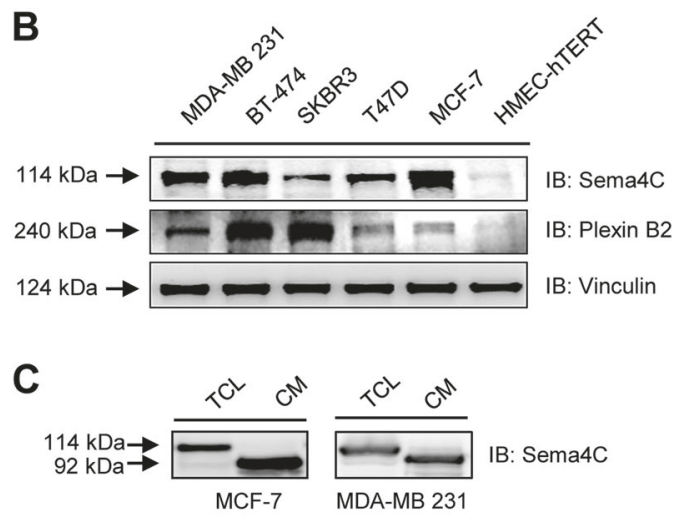

blot analysis of Sema4C and PlexinB2 expression in various breast cancer cell lines and immortalized human mammary epithelial cell line HMEC- hTERT; vinculin served as protein loading control. c Western blot analysis revealing full-length and cleaved form of Sema4C in total cell lysate (TCL) and conditioned medium (CM), respectively, of MCF-7 and MDA-MB-231 breast cancer cells

subsequent onset of cell senescence. Furthermore, by coculturing two populations of Sema4C-depleted cells labeled with different fluorescent dyes, we ruled out that multinucleated cells are the product of syncytial fusions of individual cells (Suppl. Figure 4C, D, E). Of note, there was a significant increase in nuclear levels of both p53 and p21 tumor suppressors in MCF-7 cells (Fig. 3i). Expectedly, upon Sema4C/PlexinB2 interference, the highly invasive MDA-MB-231 carcinoma cells underwent a dramatic reduction of migration and invasion ability (Suppl. Figure 5).

\section{Sema4C/PlexinB2/LARG-dependent RhoA signaling is required to sustain breast cancer cell proliferation}

The intracellular domain of PlexinB2 can elicit RhoA activation in response to ligand stimulation, through the activity of plexin-associated Rho-GEFs, such as p190-PRG and LARG [14, 18, 31, 32]. Indeed, we observed a reduced content of F-actin stress fibers in Sema4C- or PlexinB2depleted cells compared with controls (data not shown), which could be consistent with decreased RhoA activity. Actually, a recent study showed that PlexinB2 function in normal developing or adult tissues is independent of the sequence known to recruit Rho-GEFs and trigger RhoA activation [16, 33]; however, this issue has not been investigated in tumor cells. Notably, in addition to controlling the cytoskeleton, RhoA is also crucial for cell cycle progression; and inhibiting the effector Rho-associated kinase (ROCK) indeed curbed CCNA2 and CDC2 (CDK1) levels and resulted in cellular senescence [29]. Conversely, 
A

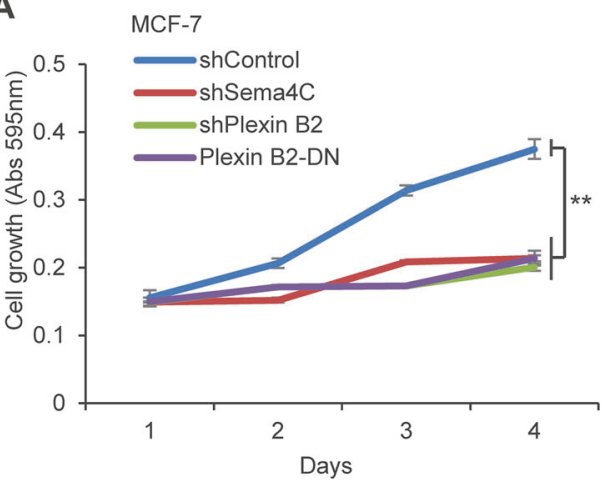

C

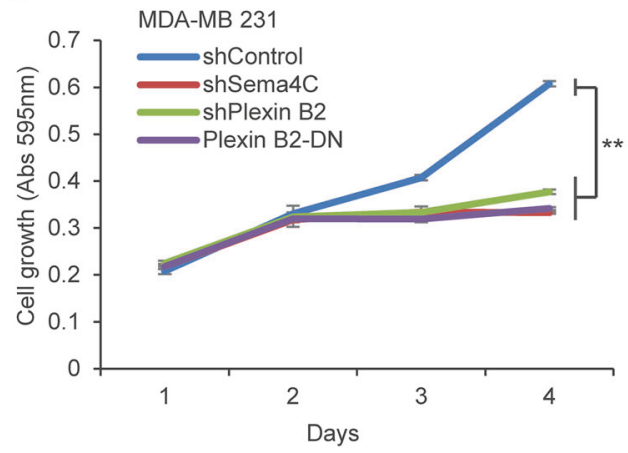

E

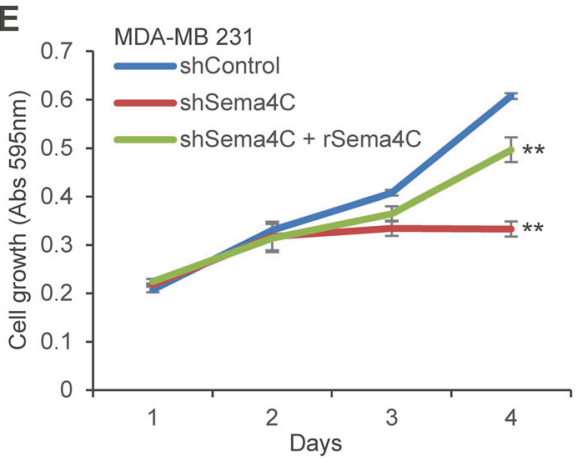

Fig. 2 Sema4C/PlexinB2 signaling blockade in breast cancer cells causes growth arrest. a-c Graphic representation of the growth curves of the indicated breast cancer cell lines (MCF-7, SKBR-3 and MDAMB-231) upon knock-down of Sema4C or PlexinB2 (by targeted shRNA), or overexpression of a PlexinB2 dominant-negative construct (B2-DN). The cells were stained with crystal violet and absorbance

RhoA activity has long been considered a trigger of breast cancer cells proliferation and metastasis [23, 24, 34, 35]. Thus, we postulated that the unexpected phenotype seen in Sema4C/PlexinB2-depleted cancer cells could be associated with reduced RhoA signaling. Indeed, consistent with previous literature [29], the inhibition of either LARG or the RhoA-effector kinase ROCK produced the same changes observed in breast cancer cells deprived of Sema4C/PlexinB2 signaling (Figs. 4a, b), including increased nuclear levels of p53 and p21 (Supp. Figure 6),
B

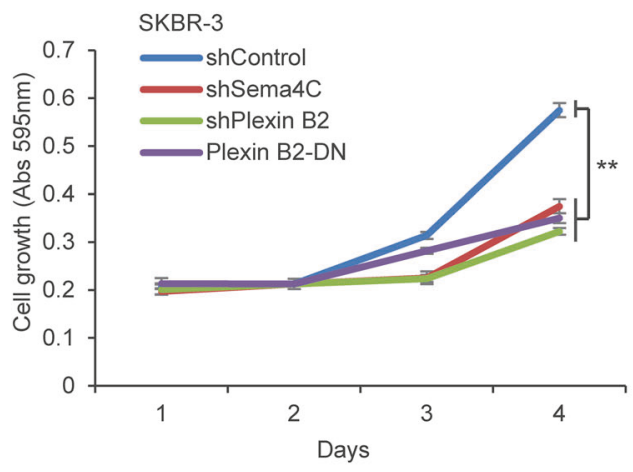

D

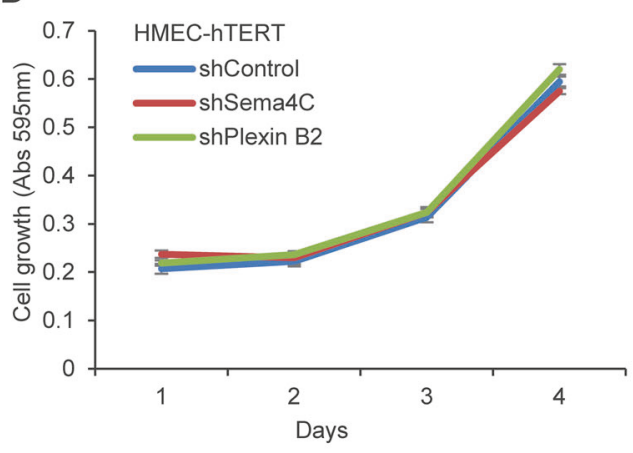

$\mathbf{F}$

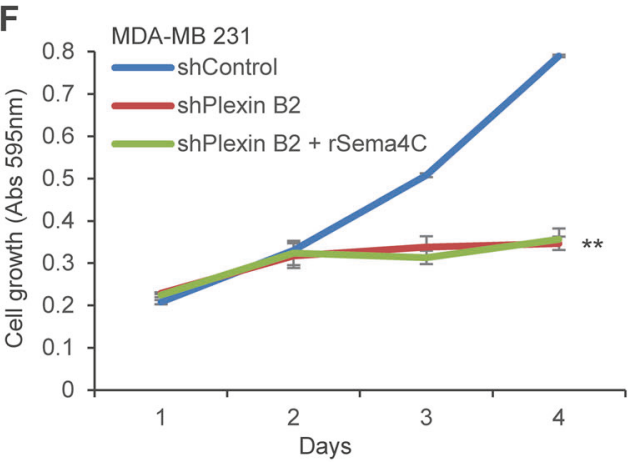

was read at $595 \mathrm{~nm}$. d Graphic representation of HMEC-hTERT cell growth curves upon silencing either Sema4C or PlexinB2 (by targeted shRNA). e, f MDA-MB-231 growth curves, upon silencing Sema4C (panel e) or PlexinB2 (panel f) in presence or absence of recombinant soluble Sema4C $(1 \mu \mathrm{g} / \mathrm{ml})$. Data are the mean \pm SD from three separate experiments; $* * p<0.01$

and long-term growth inhibition (Fig. 4c). Consistent with our mechanistic hypothesis, we demonstrated remarkable reduction of active GTP-bound RhoA levels upon silencing Sema4C or PlexinB2, or overexpressing PlexinB2 dominant-negative form (Fig. 4d). We ultimately demonstrated that defective RhoA signaling is responsible for the PlexinB2-dependent growth phenotype by rescue experiments with a constitutively active GFP-RhoA Q63L construct, which recovered both CDC2(CDK1) expression and cell proliferation in PlexinB2-deficient cells (Figs. 4e, f). 

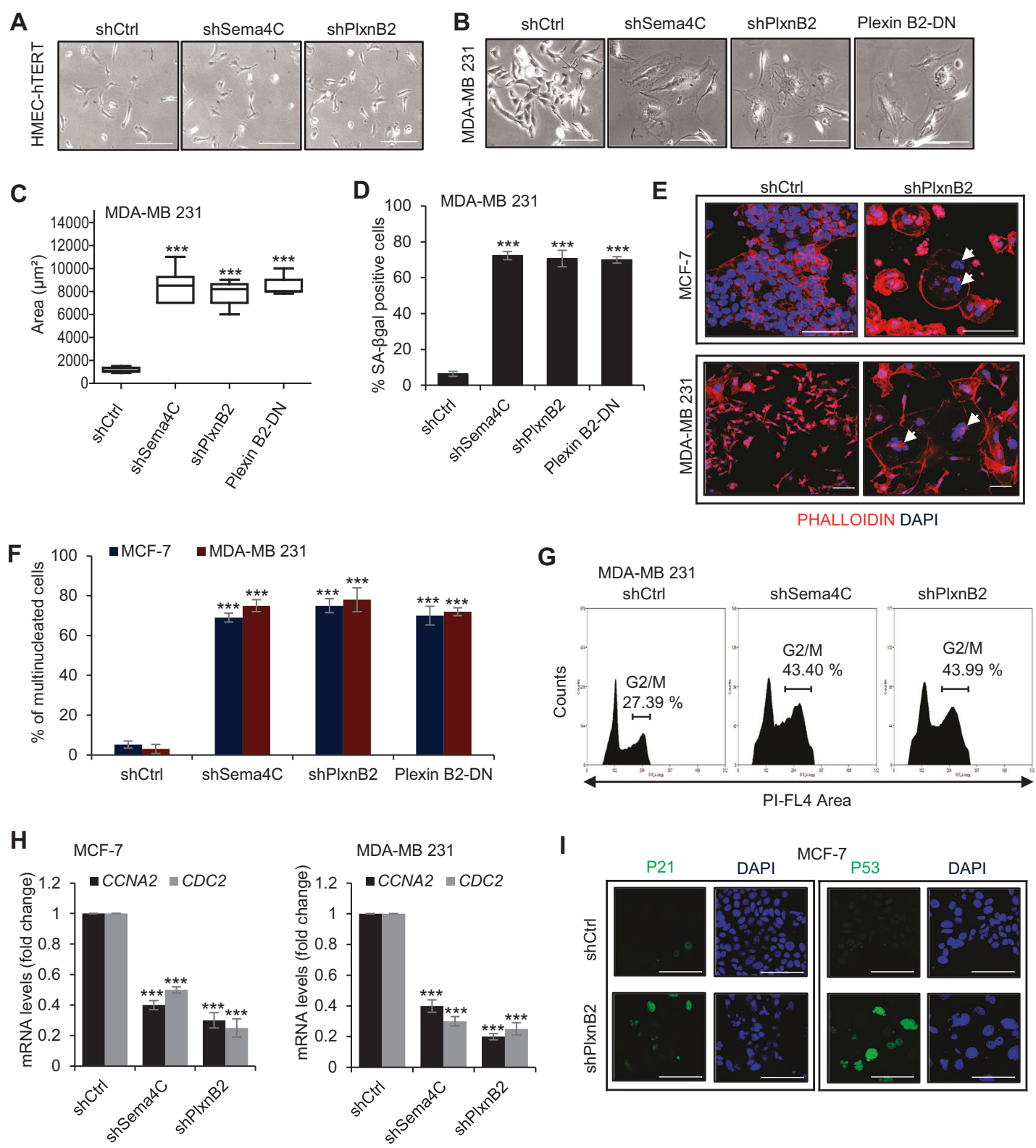

Fig. 3 Sema4C/PlexinB2 signaling blockade in breast cancer cells causes cell senescence, cell cycle inhibition and cytokinesis defects. a Bright field images of HMEC-hTERT cells subjected to Sema4C or PlexinB2 silencing (or controls). b Bright field image of MDA-MB231 cells either subjected to Sema4C or PlexinB2 silencing, or overexpressing PlexinB2-DN, or controls. Scale bar: $100 \mu \mathrm{m}$. c Quantification of area covered by engineered MDA-MB-231 described in panel b. d Fraction of SA- $\beta$-gal-positive senescent cells in the population of engineered MDA- MB-231 described in panel b. e Representative images of immunofluorescent staining of control (shCtrl) or PlexinB2-silenced (shPlxnB2) MCF-7 and MDA-MB-231 cells (as indicated), stained with Phalloidin to reveal F-actin (in red), and DAPI for nuclei (blue). f The percentage of multinucleated MCF-7 and

MDA-MB-231 cells described in panel e was assessed by computerassisted analysis of microscopic images. $\mathbf{g}$ Cytofluorimetric analysis of propidium iodide-stained MDA-MB-231 cells, either silenced for Sema4C or PlexinB2, showing relative accumulation in G2/M phase, compared with controls. h Quantitative RT-PCR analysis of mRNA levels of cell cycle genes CCNA2 and CDC2(CDK1) in MCF-7 and MDA-MB-231 cells stably silenced for Sema4C or PlexinB2 (fold change vs. control cells). i Immunofluorescence staining of p21 and p53 tumor-suppressor protein (green) revealing increased nuclear localization in MCF-7 cells depleted of PlexinB2, compared with controls; nuclei were stained with DAPI (blue). Data are the mean \pm SD from three separate experiments; $* * * p<0.001$. Scale bar: $100 \mu \mathrm{m}$. 
A

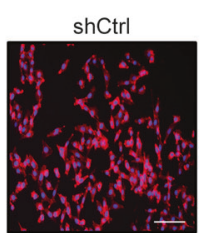

DMSO

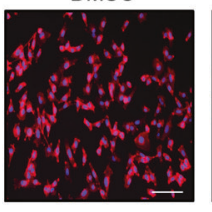

Y-16

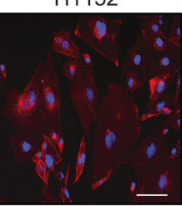

PHALLOIDIN DAPI
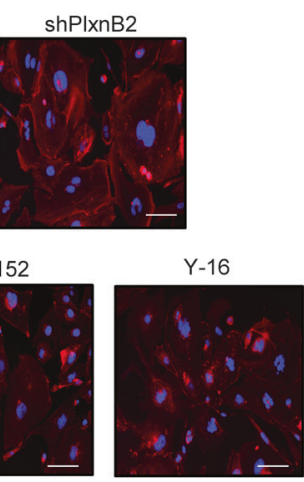

B

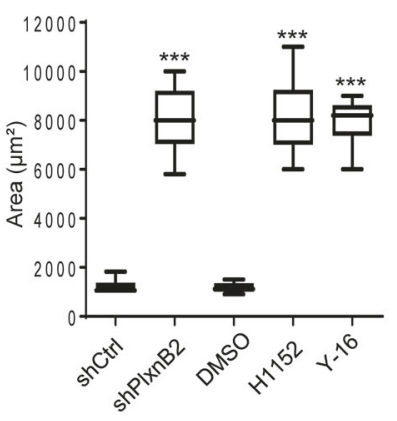

C

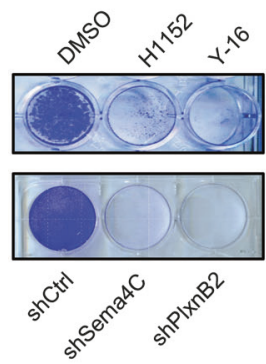

$$
\text { D }
$$

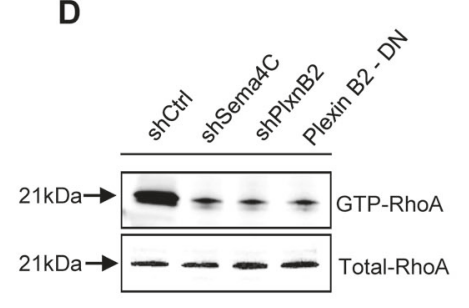

E

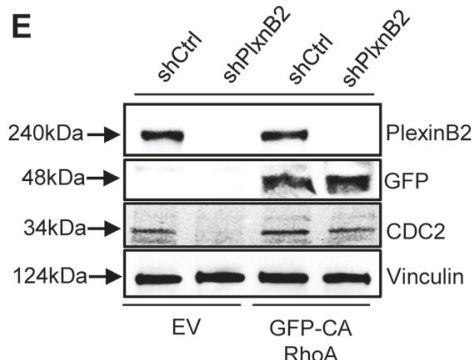

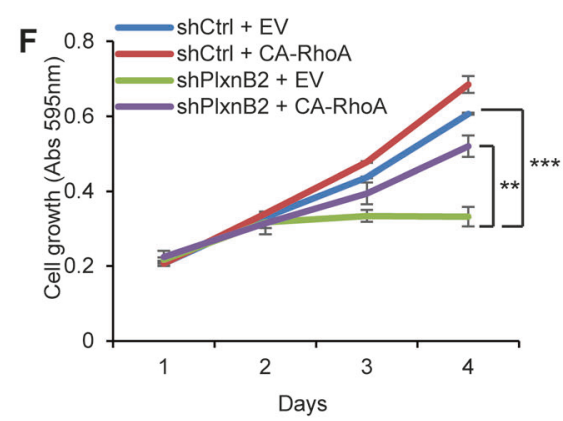

Fig. 4 Sema4C/PlexinB2/LARG-dependent RhoA signaling is required to sustain breast cancer cell proliferation. a MDA-MB-231 cells either control or subjected to PlexinB2 silencing, or treated with ROCK kinase inhibitor H1152 $(10 \mu \mathrm{M})$ or LARG inhibitor Y-16 (20 $\mu \mathrm{M})$ for 5 days, were analyzed by immunofluorescence to reveal Factin (with Phalloidin, in red) and the nuclei (with DAPI, in blue). Representative images are shown; scale bar: $100 \mu \mathrm{m}$. b Quantification of individual cell area of MDA-MB-231 cells treated as described in panel $\mathbf{a}$, by computer-assisted analysis of microscopic images. $\mathbf{c}$ Longterm colony formation assay (10 days) of parental MDA-MB-231 cells treated with $10 \mu \mathrm{M} \mathrm{H} 1152$, or $20 \mu \mathrm{M} \mathrm{Y}-16$, or with vehicle alone (DMSO); or the same cells subjected to stable silencing of Sema4C (shSema4C), or PlexinB2 (shPlxnB2), or shCtrl control cells. Fixed cells were eventually stained with crystal violet. Each condition was

\section{Enhanced Sema4C signaling disrupts cell polarity and reprograms the phenotype of luminal breast cancer cells in RhoA- and ErbB2-dependent manner}

Based on our gene knock-down experiments, Sema4C/ PlexinB2 expression appears to be crucial for breast cancer cell growth; however, the correlation between particularly elevated Sema4C levels and reduced patient survival suggested additional functions in promoting tumor progression. Intriguingly, we noticed that such correlation was particularly significant among estrogen receptor positive (ER+) carcinomas (Fig. 5a). These tumors are typically more differentiated, responsive to hormonal deprivation and rarely metastatic; yet, a small (and poorly predictable) subset of $\mathrm{ER}+$ tumors displays dramatically different outcome, due to drug resistance and metastatic dissemination. Thus, we posited that Sema4C overexpression might be a driver of repeated in triplicate; representative images are shown. d Immunoblotting analysis of active RhoA-GTP pulled-down from lysates of MDA-MB-231 cells silenced for Sema4C or PlexinB2 or overexpressing PlexinB2-DN, or controls. Detection of total RhoA in input lysates served as a loading control. e Immunoblotting analysis of PlexinB2, CDC2 (CDK1) expression in MDA- MB-231 cells silenced for PlexinB2 (or controls), upon overexpression of constitutively active (CA) RhoA (GFP- Q63L). The expression of CA-RhoA was assessed with anti-GFP antibodies. $f$ Graphic representation of MDAMB-231 cell growth curves upon silencing PlexinB2, with and without overexpressing CA-RhoA construct (same cells described in panel e). Data are the mean \pm SD from three separate experiments; $* * p<0.01$, $* * * p<0.001$

phenotypic change in this tumor subtype. To test this hypothesis, we enhanced Sema4C expression in relatively indolent luminal-type breast cancer cells MCF-7. We furthermore compared the activity of full-length transmembrane Sema4C and soluble secreted isoform comprising the sole extracellular domain (Sema4C-secr), similar to the spontaneously shed proteolytic fragment (Fig. 5b). Interestingly, we did not observe any significant increase in the proliferation rate of Sema4C-overexpressing cells in culture (Fig. 5c); however, the cellular phenotype was dramatically changed (Fig. 5d). In fact, whereas MCF-7 basally display a cobblestone phenotype, Sema4C overexpression induced fibroblast-like features and cell scattering. Notably, this was evident upon overexpressing either Sema4C full-length or Sema4C-Fc secreted fragment (Fig. 5d), indicating that the extracellular domain of the semaphorin is sufficient to elicit this effect, and pointing to a forward signaling mechanism, 
A

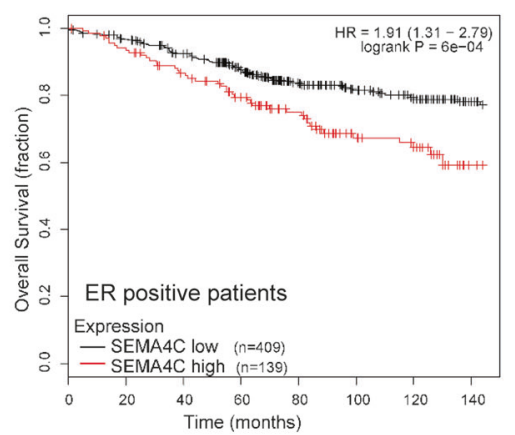

D
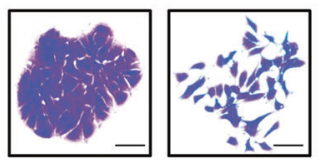

Sema4C-secr

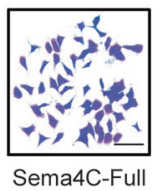

G

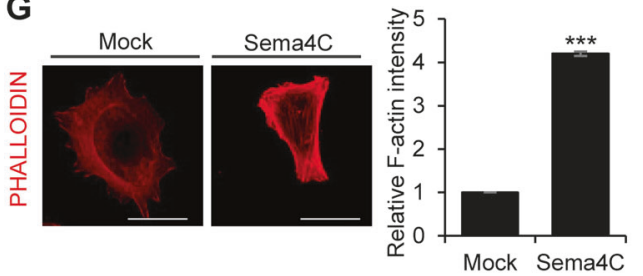

B

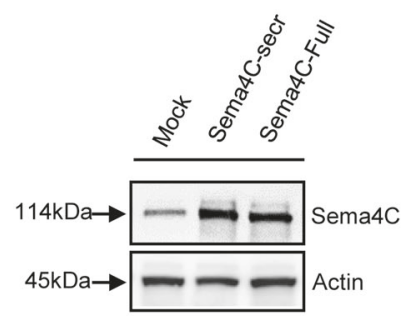

E

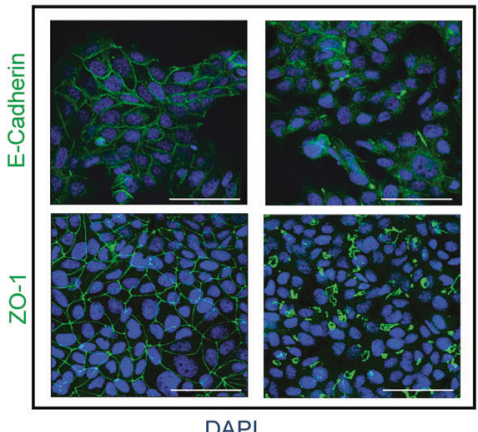

H

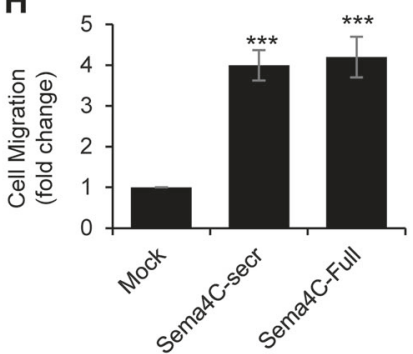

C
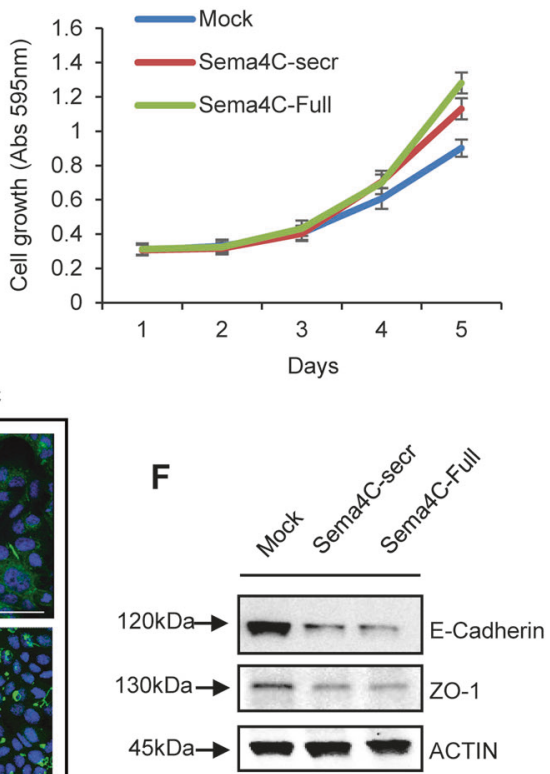

I

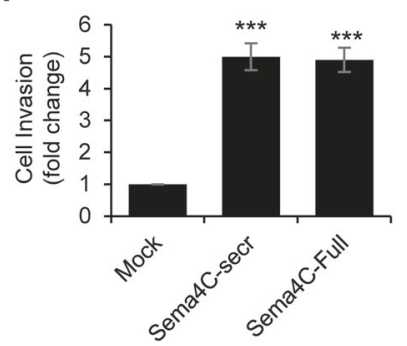

Fig. 5 Enhanced Sema4C signaling disrupts cell polarity and converts luminal breast cancer cells to acquire mesenchymal features. a Kaplan-Meier analysis of overall survival of patients (OS; follow-up at 12 years) bearing estrogen receptor positive $(\mathrm{ER}+)$ tumors $(n=$ 548) correlation to Semaphorin 4C (Sema4C) high and low expression subgroups, based on online dataset analysis by http://kmplot.com; $p=$ 6e-04. b Immunoblotting images revealing the expression of Sema4Csecr and Sema4C-full constructs in gene-transduced MCF-7 cells. c Graphic representation of MCF-7 cell growth curves, upon overexpression of Sema4C-secreted or full-length isoforms compared with Mock cells. The cells were stained with crystal violet and absorbance was read at $595 \mathrm{~nm}$. d Microscopy images of MCF-7 described above stained with crystal violet. Mock-transduced cells display a cobblestone phenotype, whereas Sema4C-overexpressing cells show an elongated fibroblast-like morphology. Scale bars: $100 \mu \mathrm{m}$. e

putatively mediated by the receptor PlexinB2. Notably, Sema4C overexpression did not affect PlexinB2 levels in cancer cells (Suppl. Figure 7A); instead, by cell immunostaining and western blots, we observed reduced expression of both ZO-1-based tight junctions and E-cadherin-based adherent junctions (Figs. 5e, f). Intriguingly, Sema4D, a close relative of Sema4C, was found to elicit ErbB2 kinase phosphorylation in cancer cells $[36,37]$ and ErbB2 kinase can regulate cell polarity complexes [38]. Indeed, in
Representative immunofluorescence images showing the expression of E-cadherin or ZO-1 (in green) in Sema4C secr-overexpressing MCF-7 cells, compared with controls; nuclei were visualized with DAPI (in blue). Scale bars: $100 \mu \mathrm{m}$. f Immunoblotting analysis of E-cadherin and ZO-1 protein expression, in MCF-7 cells overexpressing Sema4Csecreted or full-length isoforms (same as in panel d). g Immunofluorescence analysis of F-actin (with Phalloidin staining) in Mock and Sema4C secr-overexpressing MCF-7 cells. Scale bars: $20 \mu \mathrm{m}$. On the right panel, the graph on the right shows the quantification of fluorescent signal from F-actin Phalloidin staining. h, i Graphic representation of the migratory (h) and invasive (i) capability of stably transfected MCF-7 cells expressing Sema4C-secr or full-length (or Mock), assessed using transwell chamber inserts. Data are the mean \pm $\mathrm{SD}$ from three separate experiments; $* * * p<0.001$

response to recombinant purified Sema4C, ErbB2 phosphorylation on tyrosine 1248 was increased (Fig. 6a). This activity was confirmed in dose-response experiments, which also demonstrated that PlexinB2-ErbB2 kinase association predates Sema4C stimulation (Suppl. Figure 7B, C). Moreover, upon Sema4C stimulation, we found increased association between ErbB2 and PKC-Zeta, and a concomitantly decreased Par3/PKC-Zeta coupling (Fig. 6b), indicating the disassembly of Par3 polarity complexes. 

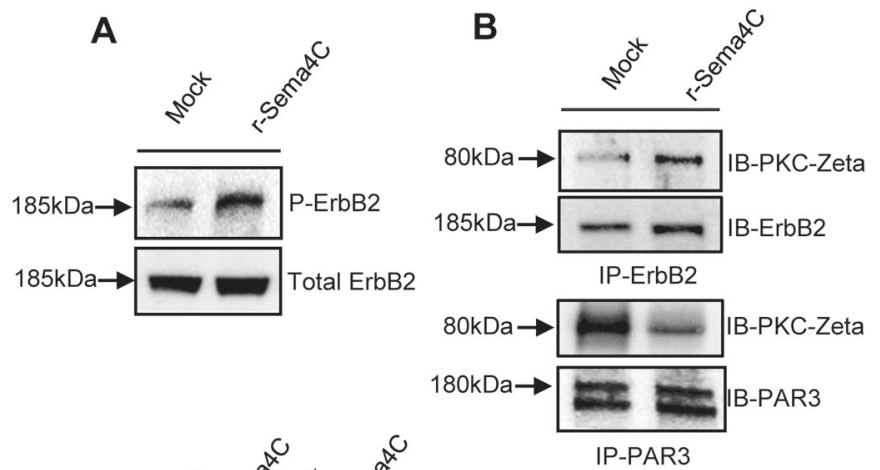
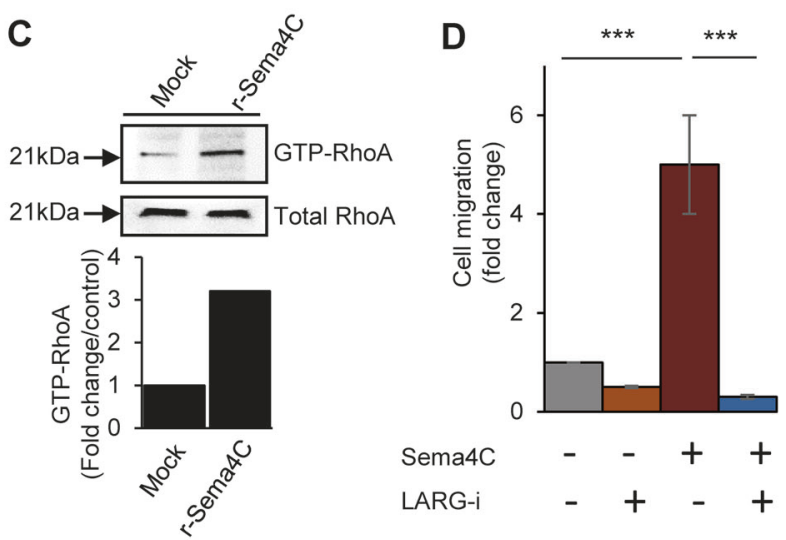

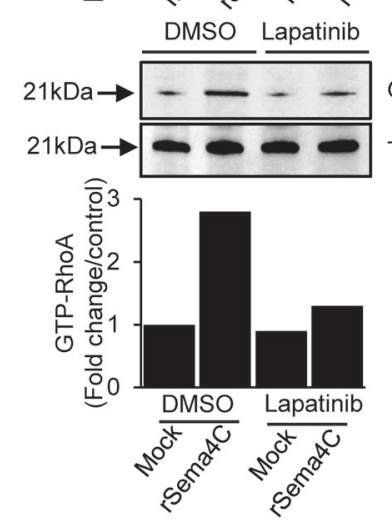

G

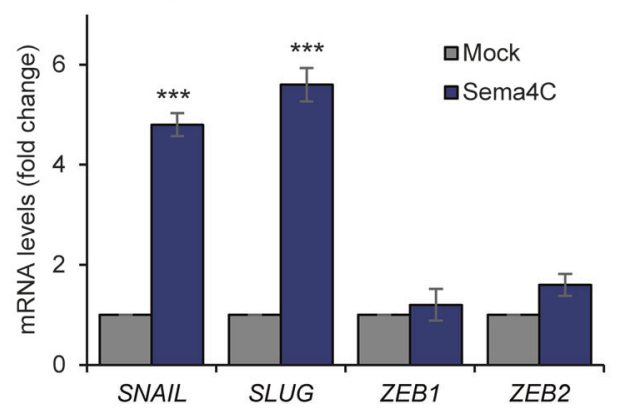

H

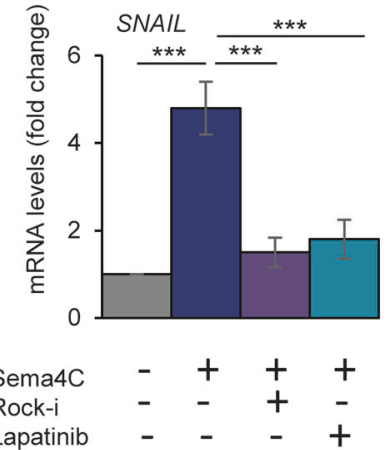

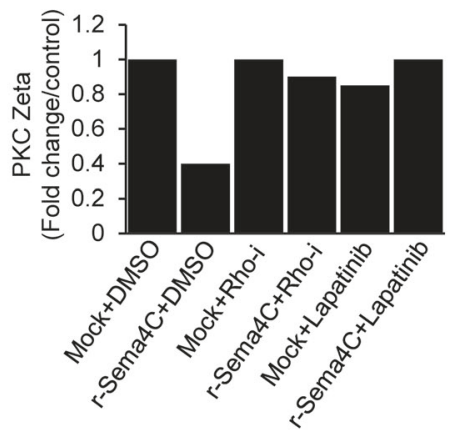

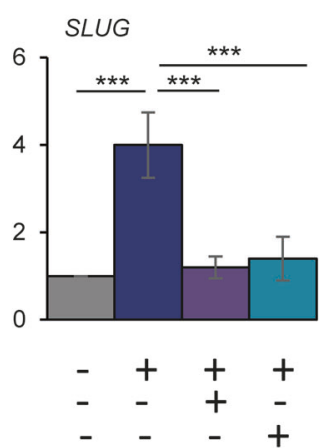

Fig. 6 Enhanced Sema4C signaling disrupts cell polarity and reprograms luminal breast cancer cells in RhoA- and ErbB2-dependent manner. a Immunoblotting analysis revealing tyrosine phosphorylated ErbB2 in MCF-7 cells upon stimulation with recombinant Sema4C $(300 \mathrm{ng} / \mathrm{ml})$ or mock, for $20 \mathrm{~min}$; total ErbB2 staining provided an internal reference. b MCF-7 cells were treated with recombinant Sema4C $(300 \mathrm{ng} / \mathrm{ml})$ or mock, for $20 \mathrm{~min}$ and protein lysates were immunopurified with anti-ErbB2 (upper panel) or anti-PAR-3 (lower panel) antibodies, followed by immunoblotting to reveal the interaction between PKC-Zeta and either of the two alternative partners. c GTP- bound active RhoA was pulled-down from lysates of serumstarved MCF-7 cells stimulated with recombinant Sema4C (300 ng/ml) for $20 \mathrm{~min}$, and revealed by immunoblotting; detection of total RhoA served as an internal reference. d Graphic representation of transwell migration efficiency (fold changes, over a period of $16 \mathrm{~h}$ ) of MCF-7 cell expressing Sema4C-secr (or mock), in the presence of the LARG inhibitor Y-16 $(20 \mu \mathrm{M})$ or DMSO vehicle alone. e Serum-starved MCF-7 cells were incubated with or without the ErbB2 inhibitor lapatinib $(10 \mu \mathrm{M})$ for $2 \mathrm{~h}$ prior to stimulation with $300 \mathrm{ng} / \mathrm{ml} \mathrm{Sema4C}$ for $20 \mathrm{~min}$. GTP-bound active RhoA was pulled-down from lysates and revealed by immunoblotting; detection of total RhoA provided an internal reference. f Serum-starved MCF-7 cells were incubated with or without the Rho-kinase Inhibitor Y-27632 $(10 \mu \mathrm{M})$ for $30 \mathrm{~min}$ or with $10 \mu \mathrm{M}$ Lapatinib for $2 \mathrm{~h}$, or with DMSO, prior to stimulation with $300 \mathrm{ng} / \mathrm{ml} \mathrm{Sema} 4 \mathrm{C}$ for $20 \mathrm{~min}$. Cell lysates were then immunopurified with anti-PAR-3 antibody (as in panel b) and the immunocomplexes were analyzed by immunoblotting with anti-aPKC and anti-PAR-3 antibodies. $\mathrm{g}$ Graphic representation of the fold change in mRNA levels of SNAIL, SLUG, ZEB1 and ZEB2 genes in MCF-7 cells stably expressing Sema4C-secr, compared with control cells, as determined by qRT-PCR analysis. $\mathbf{h}$ Graphic representation of the fold change in SNAIL and SLUG mRNA levels in Sema4C-secr-overexpressing (or control) cells treated with $10 \mu \mathrm{M}$ Lapatinib or the ROCK kinase inhibitor H1152 $(10 \mu \mathrm{M})$ for $24 \mathrm{~h}$, as determined by qRT-PCR analysis. Data are the mean \pm SD from three separate experiments; based on statistical analysis, $* * * p<0.001$ 
Sema4C-overexpressing cells also showed significantly increased F-actin stress fibers compared with mocktransfected cells (Fig. 5g), as well as greater migration and invasive properties (Figs. 5h, i). Moreover, we detected a Sema4C-dependent increase of RhoA-GTP levels (Fig. 6c), which is consistent with the complementary effect observed upon gene silencing. In addition, the treatment with the LARG inhibitor Y-16 curbed Sema4C-induced cell migration (Fig. 6d), indicating the importance of this RhoGEF in the pathway. Recently, it was also reported that RhoA-dependent kinase ROCK can phosphorylate PAR-3 and disrupt the PAR polarity complex [39]. Thus, both ErbB2 and Rho-kinase activation could disrupt cancer cell polarity. Actually, by treating with the kinase inhibitor lapatinib, we demonstrated that Sema4C-induced RhoA activation depends on ErbB2 activity (Fig. 6e). It is thus conceivable that the two effectors act in cascade to cause loss of polarity in Sema4C-overexpressing cells. Notably, the treatment with either lapatinib or the Rho-kinase inhibitor Y-27632 could prevent the dissociation of PAR-3 and PKC-zeta components of the polarity complex induced by Sema4C stimulation (Fig. 6f). Moreover, both ErbB2 and ROCK inhibition reversed the loss of E-cadherin and betacatenin-positive adherent junctions associated with Sema4C overexpression (Suppl. Figure 8A, B).

Signaling pathways sustaining a persistent EMT often converge onto transcription factors such as Snail, Slug, Twist, Zeb1 and Zeb2 [40-42]. Indeed, qPCR analysis demonstrated that Snail and Slug mRNA levels were increased upon Sema4C overexpression (Fig. 6g). Moreover, cell treatment with Rho-kinase or ErbB2 inhibitors significantly reversed Snail and Slug induction by Sema4C (Fig. 6h).

\section{Sema4C-overexpressing cells attain altered mitotic spindle orientation, detachment from the substrate and growth in suspension}

Cell polarity is a major mechanism controlling epithelial cell division and mitotic spindle orientation [43]. In the proliferation of normal epithelial sheets, the mitotic spindle is oriented parallel to the substrate, whereas a disassembly of cell polarity complexes and of the associated adherent junctions is required to allow cell detachment from the monolayer and migration/invasion in a three-dimensional context [44]. Notably, Sema4C-overexpressing MCF-7 cells, upon reaching confluency, did not show contact inhibition and formed multiple multicellular aggregates growing on top of the monolayer (Fig. 7a). By staining mitotic spindle markers alpha and gamma-tubulin, we demonstrated that whereas spindles were normally oriented parallel to the substrate in control cells, the majority of Sema4C-overexpressing cells had tilted spindle orientation, oblique or perpendicular to the substrate (Figs. 7b, c). As a consequence, one of the daughter cells for each division was placed out of the monolayer, which fostered overgrowth in multicellular aggregates, eventually released into the culture medium; importantly, detached cells did not die, but continued to grow in suspension (data not shown). This phenotype somehow reminded stem cells features, such as the ability to form spheroids in non-adhesive conditions [45]. Indeed, Sema4C overexpression induced mammosphere formation by MCF-7 cancer cells (Fig. 7d) and upregulated stem cell markers SOX-2 and Aldh3A1 (Fig. 7e). Conversely, the impairment of Sema4C/PlexinB2 signaling downregulated SOX-2 and Aldh3A1 expression (Fig. 7f), and inhibited mammosphere formation (Fig. 7g).

\section{Sema4C overexpression in luminal-type breast cancer cells leads to tamoxifen resistance, estrogen- independent growth and metastatic tumor formation in vivo}

Another typical feature of luminal-type breast cancer cells such as MCF-7 is the significant expression of steroid hormone receptors and the dependence on estrogenic stimulation, which is the base for treating ER+ tumors in the clinics with estrogen signaling inhibitors. Notably, we noticed that among ER+ patients treated with hormonal therapy, those carrying tumors with high Sema4C levels were characterized by shorter disease-free survival, potentially suggesting a relative refractoriness to target therapy (Suppl. Fig. 9). Thus, we wondered whether the Sema4Cinduced phenotypic conversion of luminal-type breast cancer cells may include a shift to estrogen independence. Intriguingly, the growth rate of Sema4C-overexpressing cells is not significantly changed in normal conditions; however, upon switching to estrogen-depleted medium, whereas control MCF-7 cells hardly proliferated, Sema4C overexpression sustained growth (Fig. 8a). Sema4C furthermore induced relative cell refractoriness to the ER signaling inhibitor tamoxifen (Fig. 8b). Estrogen-independent growth and tamoxifen resistance are often, although not always, associated with loss of ER expression in luminaltype tumor cells [46-48]. Intriguingly, we did not observe any significant difference in the mRNA levels of ER-alpha or ER-beta in control and Sema4C-overexpressing cells (Fig. 8c); however, we found reduced ER-alpha protein expression and localization in the nucleus of Sema4Coverexpressing cells (Figs. 8d, e), indicating reduced functional activity. In fact, the expression of the progesterone receptor (PR), a well-known ER target gene [49, 50], was strongly decreased in Sema4C-overexpressing cells (Fig. 8f). Moreover, we found that Sema4C and PGR levels 
A

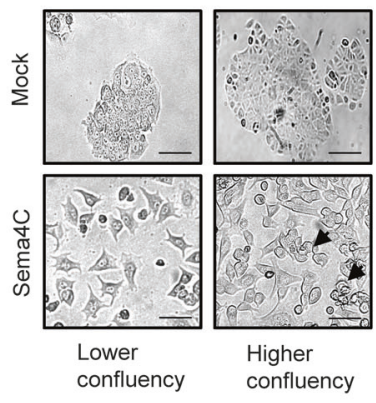

B
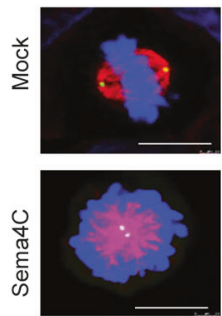

Alpha-Tubulin Gamma-Tubulin DAPI
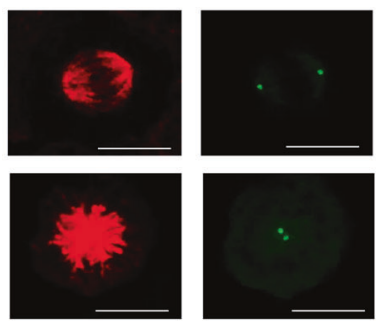

$\underline{-}$
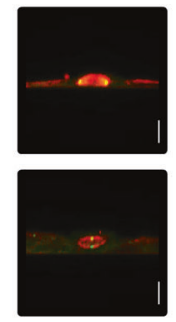

Z-stack

C

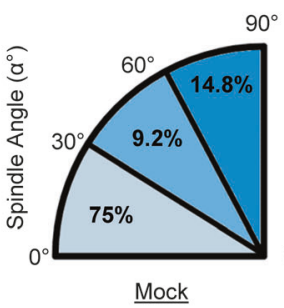

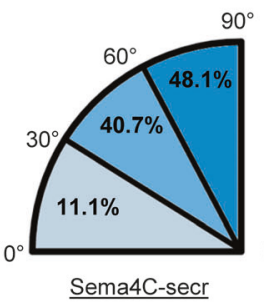

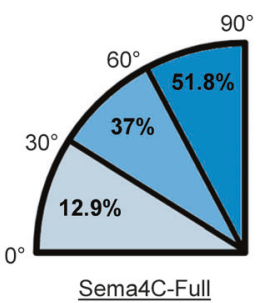

E

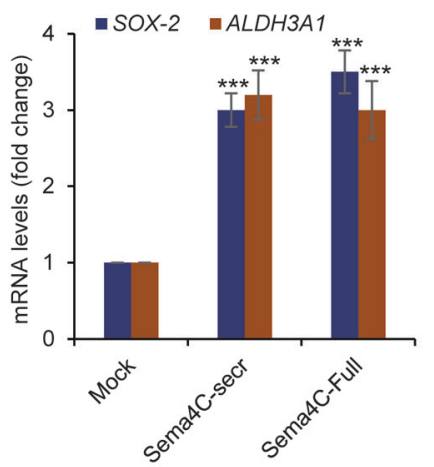

$\mathbf{F}$
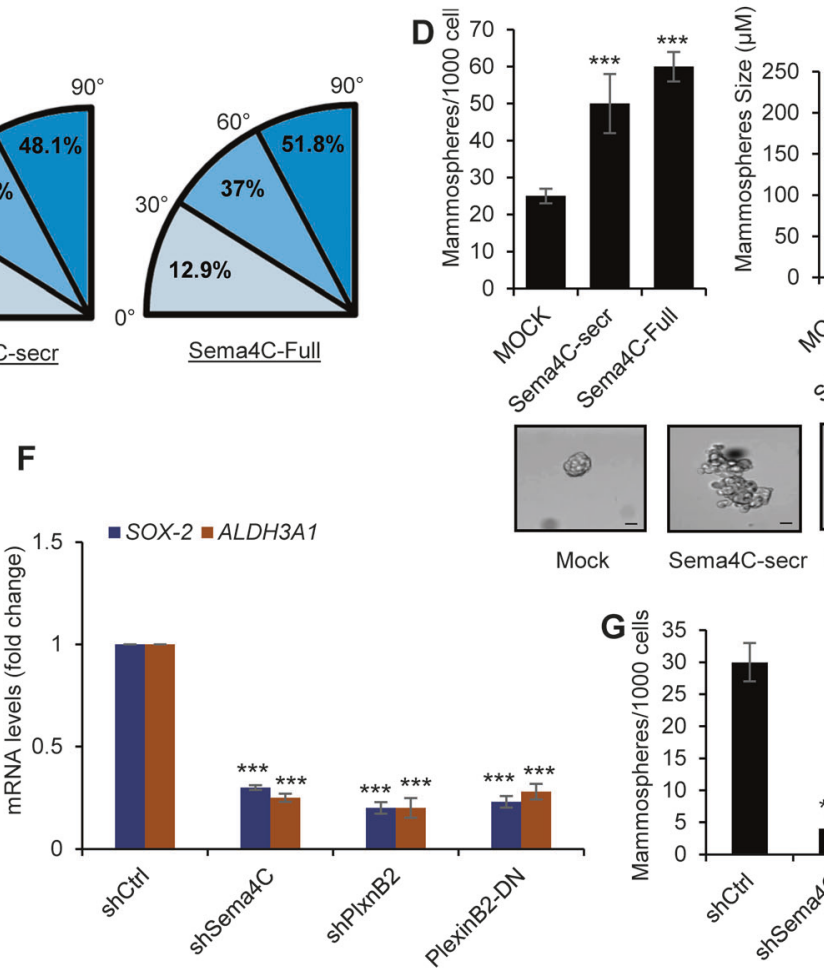

Sema4C-secr Sema4C-Full

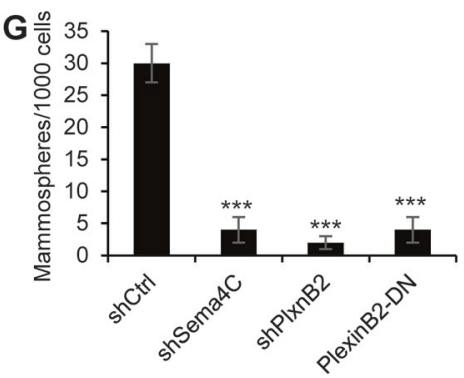

Fig. 7 Sema4C-overexpressing cells attain altered mitotic spindle orientation, detachment from the substrate and growth in suspension. a Bright field images revealing the morphology of MCF-7 mock or Sema4C-secr-overexpressing cells, in lower and higher confluency conditions. Scale bars: $100 \mu \mathrm{m}$. b Representative immunofluorescence analysis of Sema4C-secr-overexpressing (or Mock) MCF-7 cells, stained for alpha-tubulin (in red), gamma-tubulin (in green) and DAPI (blue). The rightmost panel shows corresponding $X-Z$ axis stack projections generated from laser-scanning confocal images taken at $0.5-\mu \mathrm{m}$ steps. Confocal micrographs display parallel orientation of the mitotic spindle (relative to the substrate) in Mock-transduced MCF-7 cells and a misoriented spindle in Sema4C-secr-overexpressing cell, with axis perpendicular rather than parallel to the substrate. Scale bars: $10 \mu \mathrm{m}$. c Graphical representation of distribution of mitotic spindle angles of MCF-7 cells (see Materials and methods for details), upon expression of Sema4C-secr or Sema4C full-length, or mocktransduced $(n>50$ cells in three independent experiments). d Relative frequency and average size of mammospheres formed by MCF-7 cells upon Sema4C-secr or Sema4C full-length expression, or controls. Below the graph shown are representative bright field images of the different conditions; scale bars: $20 \mu \mathrm{m}$. e qRT-PCR analysis of mRNA expression of stemness markers SOX-2 and ALDH3A1, in Sema4Csecr and Sema4C full-length expressing MCF-7 cells, compared with Mock. f qRT-PCR analysis of mRNA expression of SOX-2, ALDH3A1 in MCF-7 cells upon silencing Sema4C, PlexinB2 or overexpressing PlexinB2-DN, compared with controls. g Relative frequency of mammospheres formed by MCF-7 cell silencing Sema4C, PlexinB2 or overexpressing PlexinB2-DN, compared with controls. Data are the mean \pm SD from three separate experiments; $* * * p<0.001$ are inversely correlated in large datasets of breast cancer, such as TCGA [51] (Fig. 8g).

Finally, we asked whether this novel phenotype elicited by enhanced Sema4C signaling could also foster the tumorigenic properties in vivo of MCF-7 cells, otherwise relatively indolent and known to form localized tumors in mice only upon estrogen supplementation $[52,53]$. Thus, we implanted in mice mock-transduced or Sema4C-overexpressing cells, and monitored their growth in absence of estrogen supplementation. Although control cells expectedly formed very slowly growing tumors, we observed exponential growth of xenografts formed by Sema4C-overexpressing cells, in the 
A

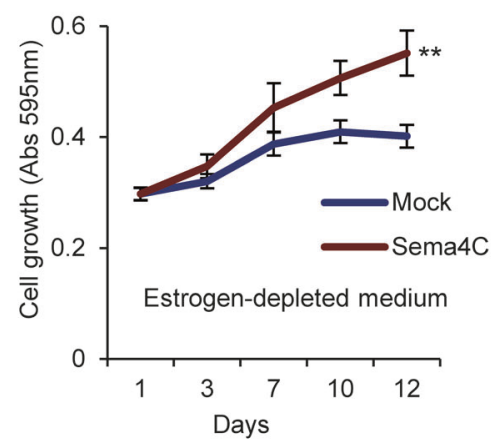

B

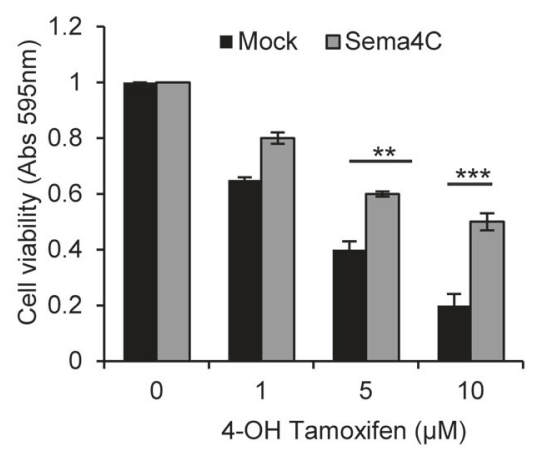

C
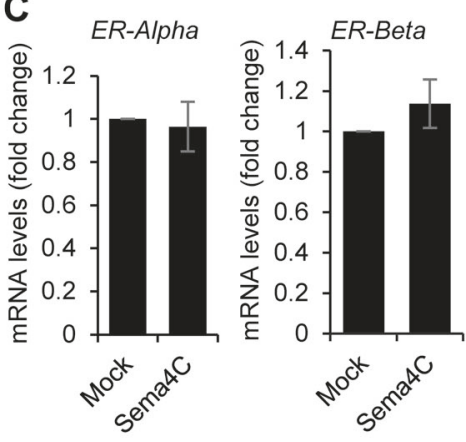

D

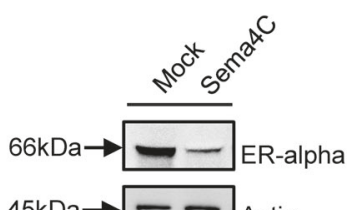

$45 \mathrm{kDa} \rightarrow--$ Actin
E

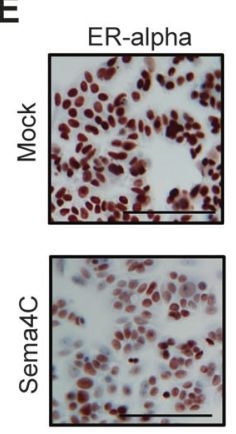

$\mathbf{F}$

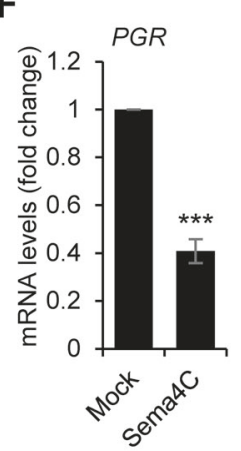

G

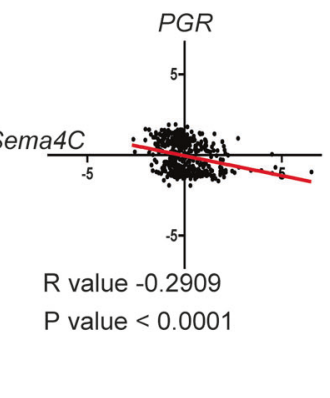

H

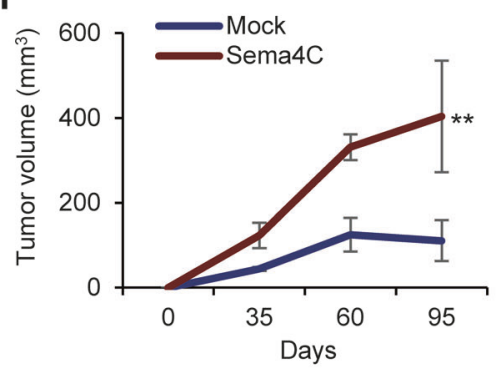

I

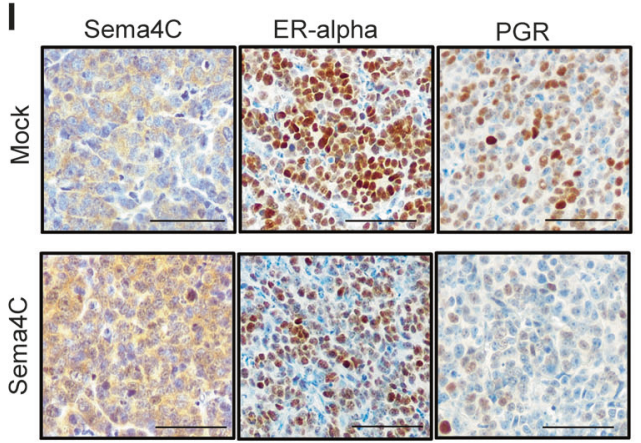

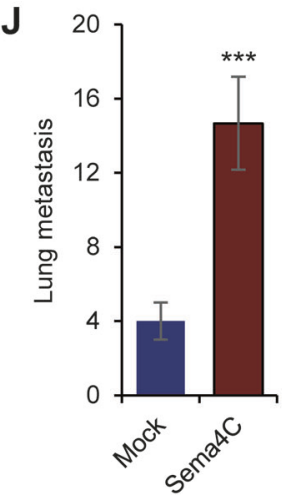

Fig. 8 Sema4C overexpression in luminal-type breast cancers cells leads to tamoxifen resistance, estrogen-independent growth and metastatic tumor formation in vivo. a Graphic representation of the growth curves of MCF-7 cells overexpressing Sema4C-secr (or Mock) in estrogens-depleted medium (phenol red-free DMEM with 5\% charcoal-stripped FBS). The cells were stained with crystal violet and absorbance was read at $595 \mathrm{~nm}$. b Graphical representation of relative fold change in the cell viability of Mock and Sema4C-secroverexpressing cell treated with the indicated increasing concentrations of 4-OH tamoxifen for 5 days (or with vehicle alone). c qRTPCR analysis of mRNA expression of estrogen receptor genes (ERalpha and ER-beta) in MCF-7 cells overexpressing Sema4C-secr, compared with Mock cells. d Immunoblotting analysis of ER-alpha protein expression in Mock and Sema4C- secr-overexpressing cells; beta-actin staining provided a protein loading control. e Immunocytochemistry analysis of ER-alpha expression and nuclear localization in Mock and Sema4C-secr-overexpressing MCF-7 cells. Scale bars:

absence of any estrogen supplementation (Fig. 8h), indicating a dramatic subversion of the behavior of these cells in vivo. In situ tumor analysis revealed reduced expression of ER-
$100 \mu \mathrm{m}$. f qRT-PCR analysis of mRNA expression of progesterone receptor (PGR) in MCF-7 cells overexpressing Sema4C-secr, compared with Mock. g Z-score correlation plot between Sema4C and progesterone receptor (PGR) expression, based on the analysis of TCGA breast cancer dataset (Breast Invasive Carcinoma, TCGA, Cell 2015-cBioPortal; $n=421$ cases); $p<0.0001$. h MCF-7 cells overexpressing Sema4C-secr (or mock-transduced) were orthotopically injected into the mammary fat pad of female nude mice $(n=5$ per condition), without any estrogen supplementation, and tumor burden was assessed at the indicated time points. i Representative immunohistochemical images showing Sema4C, ER-alpha and PGR expression in Sema4C-secr-overexpressing (and Mock) tumors described in panel h. Scale bars: $100 \mu \mathrm{m}$. j Quantification of spontaneous lung macro-metastasis detected in tumor-bearing mice described in panel $\mathbf{h}$. Data are the mean \pm SD from three separate experiments; $* * p<0.01$, $* * * p<0.001$

alpha and dramatic decrease in PRG levels in Sema4Coverexpressing tumors (Fig. 8i). Moreover, although control tumors, after long-term growth in mice, were associated with 
few occasional metastatic lesions in the lungs, Sema4C promoted the formation of a remarkable number of macrometastasis (Fig. 8j). These results strongly suggest that the acquisition of elevated Sema4C expression in luminal-type $\mathrm{ER}+$ mammary tumor cells can strikingly convert their phenotype to tamoxifen resistant, locally invasive and metastatic.

\section{Discussion}

Mice deficient for either Sema4C or its receptor PlexinB2 are embryonic lethal, due to major nervous system developmental defects $[54,55]$. Yet, these genes do not seem to be required for normal cell growth, either in the embryo or in adult tissues [16]. Actually, the molecular mechanisms of PlexinB2 signaling in different cell types remained unclear.

PlexinB2 is widely and prominently expressed in carcinoma cells; Sema4C expression instead is variably distributed in human tumors. By analyzing different datasets, we found a clear correlation between elevated Sema4C levels and poor outcome of breast cancers, which deserved further investigation. Of note, Sema4C expression is uniformly distributed across different subtypes of breast cancers (data not shown); this suggested that Sema4C-high tumors feature a novel subset characterized by poor patient survival, which does not seem to fit with classical breast cancer subtypes. Recently, recombinant Sema4C was found to promote the growth and migration of tumor and lymphatic endothelial cells, by transactivating either ErbB2 or Met tyrosine kinases [11]. In our study, we demonstrate that Sema4C/PlexinB2 signaling is specifically required for breast cancer cell proliferation, by maintaining elevated RhoA signaling. This function is dependent on the plexinassociated RhoA exchanger LARG. Targeting Sema4C/ PlexinB2 signaling resulted in cell cycle arrest in G2/M phase (due to impairment of cytokinesis), upregulation of tumor-suppressor genes and cell senescence. Notably, the expression of constitutive active RhoA could rescue these growth defects. Thus, our data indicate that, different from normal cells, RhoA activity is essential for cancer cell viability and growth, and Sema4C/PlexinB2 signaling is a crucial mechanism maintaining this function in breast cancer cells.

As a subset of breast tumors with particularly elevated Sema4C is characterized by poor outcome, we investigated the impact of Sema4C overexpression in ER+ luminal-type breast cancer cells that are normally characterized by a better prognosis. Notably, we did not observe any significant increase in cell proliferation in response to Sema4C; however, there were major RhoA-dependent functional changes in luminal-type breast cancer cells, which could account for the acquisition of an aggressive phenotype. In particular, MCF-7 cells underwent disassembly of planar polarity complexes, loss of cell-cell adhesions, and misoriented cytokinesis leading to the formation of multicellular aggregates detaching from the monolayer. From the mechanistic standpoint, this major phenotypic conversion was mediated by PlexinB2-associated effectors ErbB2 kinase and RhoA-activator LARG. Importantly, Sema4Coverexpressing MCF-7 acquired the ability to grow in estrogen-independent manner and form lung metastasis in mice. Usually, resistance to hormonal deprivation of breast cancers is associated with the loss of ERs [46-48], but Sema4C-high tumor cells were still ER+, although there was a significant decrease in ER nuclear localization. These novel findings suggest that Sema4C/PlexinB2 signaling can impair ER signaling in breast cancer cells and enable estrogen-independent growth and refractoriness to hormonal therapy. This could be particularly relevant in translational medicine, as a poorly characterized subset of ER+ luminal breast cancers is actually resistant to estrogen deprivation and can give rise to distant metastasis.

In sum, we demonstrated that Sema4C/ PlexinB2 signaling is required for the proliferation of breast cancer cells of different subtypes. Moreover, enhanced Sema4C signaling in luminal-type ER+ breast tumor cells promoted a major phenotypic and behavioral switch, including reduced sensitivity to hormonal therapy and the acquisition of invasive/metastatic properties. Importantly, as Sema4C and PlexinB2 levels are robustly increased in breast cancer cells compared with normal counterparts, molecular therapies targeting this signaling axis could be relatively tumor specific; hence, its inhibition may represent a novel therapeutic approach to curtail cancer cell growth and invasive/metastatic progression.

\section{Materials and methods}

\section{Cell lines and drugs}

Tumor cell lines were obtained from ATCC and cultured in a humidified incubator with $5 \% \mathrm{CO}_{2}$ at $37^{\circ} \mathrm{C}$ in media supplemented with $10 \%$ fetal bovine serum (FBS), $100 \mathrm{IU} /$ $\mathrm{ml}$ of penicillin $\mathrm{G}$ sodium and $100 \mu \mathrm{g} / \mathrm{ml}$ of streptomycin sulfate. HMEC-hTERT cells were cultured in 1:1 Dulbecco's modified Eagle's medium (DMEM)/Ham's F12 medium mixture, complemented with $10 \%$ FBS, $100 \mathrm{U} / \mathrm{ml}$ penicillin-streptomycin, $2 \mathrm{mM}$ L-glutamine, $10 \mathrm{ng} / \mathrm{ml}$ human epidermal growth factor (EGF), $0.5 \mu \mathrm{g} / \mathrm{ml}$ hydrocortisone and $10 \mu \mathrm{g} / \mathrm{ml}$ insulin. MDA-MB-231 and MCF-7 cells were cultured in DMEM; SKBR-3 cells were cultured in RPMI medium. For estrogen-dependent tamoxifen sensitivity studies, growth and drug assays performed in MCF- 
7 were done in phenol red-free DMEM with $0.5 \%$ or $5 \%$ charcoal-stripped serum with the addition of either vehicle (ethanol) or different concentrations of 4-OH tamoxifen (Sigma), as indicated. Cell treatments with Y-27632 (Sigma, Y0503-1MG; used $10 \mu \mathrm{M}$ or $30 \mu \mathrm{M}$ ), H1152 dihydrochloride (Tocris, $871543-07-6$ used $10 \mu \mathrm{M}$ or 30 $\mu \mathrm{M}$ ), Lapatinib (used $10 \mu \mathrm{M}$ ), Y-16 inhibitor $20 \mu \mathrm{M}$ (Sigma, SML0873) were used for the indicated times.

\section{Antibodies and other reagents}

The following antibodies from Santa Cruz were applied to detect the indicated proteins: anti-PlexinB2 (I-16, sc34504), anti-PKC-Zeta (C-20, sc-216), anti-p21 (F-5, sc6246), anti-p53 (DO-1, sc-126), anti-ErbB2 (C-18, sc-284), anti- $\beta$-tubulin (H-235, sc-9104), anti-GFP (B-2, sc-9996), anti-cdc2-p34 (B-5, sc-137035), anti-Sema4C (sc-136445) and anti-actin (sc-1616). The following antibodies were from Sigma-Aldrich: anti- $\gamma$-tubulin (T3559), anti-vinculin (V4505), anti- $\gamma$-tubulin (clone GTU-88, T6557) and antiZO-1 (SAB1306492). Additional antibodies applied in this study: anti-E-cadherin (BD-Transduction cat. 610182), antiPAR-3 (07-330, Merck Millipore), anti- $\alpha$-tubulin (ab15246, Abcam), anti-phospho-ErbB2-1248 (\#2247) and anti-ER- $\alpha$ (D8H8; \#8644, for immunoblotting) were from Cell Signaling; anti-human ER- $\alpha$ (cl. EP1, for immunohistochemistry) and anti-human PR (cl. 636) were from DAKO. Secondary antibodies were from Amersham. SA- $\beta$ galactosidase activity was detected using the Senescence $\beta$-Galactosidase Staining Kit from Cell Signaling.

\section{Gene transfer and RNA interference}

Stable complementary DNA (cDNA) and shRNA expression in mammalian cells was typically achieved by means of lentiviral vectors. Calcium phosphate method was used to co-transfect transfer plasmids, packaging vectors and constructs expressing VSV-G envelope protein in 293T cells, according to validated methods [56]. Tumor cells were transduced by incubation with lentiviral vector suspensions, in the presence of $8 \mu \mathrm{g} / \mathrm{ml}$ polybrene, for $8-12 \mathrm{~h}$. In other experiments, cDNA and small interfering RNA-expressing constructs were transiently transfected with Lipofectamine 2000 (Life Technologies) according to manufacturer's instructions. Sema4C downregulation was achieved by interference with shRNA TRCN0000060694, TRCN0000060695, TRCN0000060697 and TRCN0000060697 and PlexinB2 downregulation was achieved by interference with shRNA TRCN0000048188, TRCN0000048189, TRCN0000048190 and TRCN0000048192 by lentiviral vectors (puromycin selectable construct from Sigma Mission library). Fulllength human Sema4C (obtained from Promega-Kazusa library) and its mutant lacking transmembrane and cytoplasmic domain (Sema4C-secr) was generated by PCR with forward primer: 5'CGTTGCTAGCCATGGGACCACACTGGGCTGTC-3' and reverse primer: 5' GCAATCCGGAGCCTGCCACGACAGCCACAAGGTAG-3' and subcloned into pLVX (Invitrogen) lentiviral vector. Human PlexinB2 dominant-negative (B2-DN) consisting of extracellular and transmembrane regions (amino acid: 1-1220) was subcloned into pLVX (Invitrogen) lentiviral vector. Constitutive active GFP-RhoA Q63L was obtained from Addgene (Plasmid \#12968).

\section{RhoA-GTP pull-down assay}

The levels of active GTP-bound RhoA in cell lysates were measured by pull-down with a GST (Glutathione S-Transferase) fusion protein with the RhoA-binding domain of Rhotekin (RBD), according to manufacturer's instructions (Thermo Fischer Scientific, cat. 16116).

\section{Immunofluorescence and immunohistochemical analysis}

For immunofluorescence analysis, cells were plated on round coverslips. Cells were fixed in $4 \%$ paraformaldehyde for $15 \mathrm{~min}$, permeabilized with $0.1 \%$ Triton/phosphatebuffered saline (PBS) for $3 \mathrm{~min}$ at room temperature, and blocked by $5 \%$ normal goat serum for $30 \mathrm{~min}$. The cells were then incubated with primary antibodies for $1 \mathrm{~h}$ (or overnight), followed by incubation with the fluorochromeconjugated secondary anti-mouse or anti-rabbit $\operatorname{IgG}(\mathrm{H}+\mathrm{L})$ for $30 \mathrm{~min}$ at $37^{\circ} \mathrm{C}$. F-actin was stained by using fluorescent-Phalloidin conjugates. Nuclei where stained with 4,6-diamidino-2-phenylindole (DAPI). The coverslips were then washed and mounted on slides. Images were acquired at room temperature with a confocal laserscanning microscope (SPEII DM5500 CSQ; Leica) equipped with a $63 \times / 1.30 \mathrm{HCX}$ Plan-Apochromat oil immersion objective lens (ACS APO 63×/1.30 oil CS 0.17/E, 0.16) using Leica LAS AF software. Images were analyzed with LAS AF Lite software. For differential labeling of cells with viable dyes we used CellTrace ${ }^{\mathrm{TM}}$ CFSE (Catalog number: C34554) and Vybrant ${ }^{\circledR}$ DiD cell-labeling solution (Catalog number: V22887). For immunohistochemical analysis, paraffin-embedded or OCT (Optimal Cutting Temperature compound)-embedded frozen samples were cut in 10- $\mu \mathrm{m}$ thick sections and probed with primary antibodies. Streptavidin was conjugated with horseradish peroxidase (HRP, DAKO), whereas all secondary antibodies used were conjugated with Alexa Fluor 488 or Alexa Fluor 546 fluorochromes (Molecular Probes). Slides were analyzed 
using a Leica DM IRBM microscope, and digital images were evaluated with METAMORPH software.

\section{Cell cycle analysis}

Cultured cells ( $10^{5}$ cells per condition) were washed with PBS and trypsinized, then fixed in an ethanol (80\%)/PBS solution at $4{ }^{\circ} \mathrm{C}$ for overnight. Then, the cells were incubated in a solution containing $0.2 \mathrm{mg} / \mathrm{ml} \mathrm{RNase}$ at $37^{\circ} \mathrm{C}$ for $30 \mathrm{~min}$, and stained with $50 \mathrm{ng} / \mathrm{ml}$ propidium iodide at room temperature for $30 \mathrm{~min}$, and finally analyzed by flow cytometry (FACS caliber; Becton Dickinson, San Jose, CA).

\section{Analysis of mitotic spindle orientation}

To examine the spindle orientation in MCF-7 cells, Z-stack images were generated from $0.5 \mu \mathrm{m}$-thick sections of metaphase cells, which were immunostained with antigamma-tubulin, anti-alpha-tubulin antibodies and DAPI. The linear distance and the vertical distance between the two poles of the metaphase spindles was measured. Then, the angle between the axis of a metaphase spindle and that of the cell-adhesion surface ( $\alpha^{\circ}$, spindle angle) was calculated as described [57]. The distribution of measured spindle angles ( $n>50$, per each condition) were plotted in radial histograms.

\section{In vivo tumor models}

Animal handling was performed according to international guidelines, and it has been approved by the local Ethical Commission and by the Italian Ministry of Health. In particular, NOD/SCID female mice (Charles River Laboratories) where used in the study. In all, $2 \times 10^{6} \mathrm{MCF}-7$ cells overexpressing Sema4C and Mock cells suspended in 100 $\mu \mathrm{L}$ PBS was injected per each mice ( $n=5$ mice per condition). Tumor volume was measured with calipers for a duration of 95 days during the study. At the end of the experiment, mice were sacrificed and tumor masses and lungs were fixed in $4 \%$ paraformaldehyde and embedded in paraffin. Lung metastasis was counted after counterstaining airways with India ink.

\section{Cell proliferation assay}

Tumor cells were seeded in multiple 96-well plates at an initial density of $1.5-3 \times 10^{3}$ cells per well (depending on the cell line), and subsequently let to grow in complete culture medium with $10 \%$ FBS. Every $24 \mathrm{~h}$, one multiwell dish was fixed with $11 \%$ glutaraldehyde, stained with crystal violet and the absorbance was read using a standard colorimetric system at $595 \mathrm{~nm}$. For long-term colony formation assays, depending on the cell line, 500-3000 cells per well were seeded in a six-well plate and grown either on $10 \%$ serum or $1 \%$ serum with or without drugs and allowed to form colonies in 1-3 weeks. Colonies are fixed with glutaraldehyde $(11.0 \% \mathrm{v} / \mathrm{v})$, stained with crystal violet $(0.5 \% \mathrm{w} / \mathrm{v})$ and observed using a stereomicroscope.

\section{Protein analysis}

Total cell lysates were prepared in a Tris $\mathrm{pH} 6.8-5 \%$ sodium dodecyl sulfate solution by heating at $95{ }^{\circ} \mathrm{C}$ for 20 min. Protein concentration was measured using standard Pierce BCA protein assay. For western blotting, 10-40 $\mu \mathrm{g}$ of protein were separated in $7.5 \%$ or $10 \%$ mini gels from Bio-Rad, transferred to nitrocellulose membrane using semi-dry method, and immunoblotted according to standard protocol. For immunoprecipitation experiments, the cells were lysed in a ice cold lysis buffer (EB) containing $1 \%$ Triton $\mathrm{X}-100,10 \mathrm{mM}$ Tris, $\mathrm{pH} 7.6,50 \mathrm{mM} \mathrm{NaCl}, 0.1 \%$ bovine serum albumin (BSA) adding phosphatase and protease inhibitors: $1 \mathrm{mM}$ PMSF (Phenylmethylsulfonyl fluoride), $1 \mathrm{mM} \mathrm{Na} \mathrm{VO}_{4}, 1 \mathrm{mM} \mathrm{NaF}, 1 \mathrm{mM}$ aprotinin, 1 $\mathrm{mM}$ leupeptin and $1 \mathrm{mM}$ pepstatin. Equal amounts of cell lysates were incubated with Protein A- or Protein GSepharose beads coated with specific antibodies. After $2 \mathrm{~h}$ of incubation at $4{ }^{\circ} \mathrm{C}$, the beads were washed and immunoprecipitated proteins were denaturated with boiling in Laemmli buffer according to manufacturer's protocols.

\section{Real-time quantitative PCR analysis of gene expression}

Total RNA from tumor cell lines was isolated using RNeasy Protect Mini kit (Qiagen) according to manufacturer's instructions. cDNA preparation was performed according to standard procedures, using M-MLV Reverse Transcriptase (Promega) and oligo-dT primers/random hexamers. Quantitative real-time PCR was performed by applying the following Taqman probes (Applied Biosystems): Hs00367063_m1(PLXNB2), Hs00195591_m1(SNAI1), s01023894_m1(CDH1) and Hs00950344_m1(SNAI2). Alternatively, PCR was conducted with SYBR Green Master Mix (Life Technologies) and run in Applied Biosystems 7900HT Fast Real-Time PCR system, by applying the following primer pairs:

GAPDH (F: 5'-GAAGGTGAAGGTCGGAGTC-3', R: 5'-GAAGATGGTGATGGGATTTC-3'), Snail (F: 5'-GACTACCGCTGCTCCATTCCA-3'，R: 5'-TCCTCTTCATCACTAATGGGGCTTT-3), Zeb2 (F: 5'-TTCCATTGCTGTGGGCCT-3', R: 5'-TTGTGGGAGGGTTACTGTTGG-3'), Slug (F: 5'-AGATGCATATTCGGACCCAC-3', R: 5'-CCTCATGTTTGTGCAGGAGA-3), Zeb1 (F: 5'AGCAGTGAAAGAGAAGGGAATGC-3', R: 5'-GGTCCTCTTCAGGTGCCTCAG-3'), h-E-Cadherin (R: 5'- 
GTCACCTTCAGCCATCCTGT-3', F: 5'-GGGTTATTCCTCCCATCAGC-3), h-SOX2 (F: 5'-GCGGCAACCAGAAAAACA-3', R: 5'-TCTCCGTCTCCGACAAAAGT3), h-p21 (F: 5'-GGAAGACCATGTGGACCTGT-3', R: 5'-GGCGTTTGGAGTGGTAGAAA-3'), h-Plxn-B2 (R: 5'CTTCGTCCGCTCCTTCTCTT-3', F: 5'-CCACCCTCATCCTGTCCAA-3'), h-Plxn-B2 (R: 5'-CTTGACCTGGGAGATGGTGT-3', F: 5'-CTGGGGGATGATGTGGAGTA-3'), h-P53 (F: 5'-GCGTGTGGAGTATTTGGATG-3', R: 5'-CCAGTGTGATGATGGTGAGG-3'), hP53 F: 5'-CTTTGAGGTGCGTGTTTGTG-3', R: 5'-CTTCTTTGGCTGGGGAGAG-3'), h-Sema4C (F: 5'-GACACCTCCTGGCACAACAC-3', R: 5'-CCACTTCTGGGCTTCCTCA-3', h-beta-actin (F: 5'-GGAGGAGCTGGAAGCAGCC-3', R: 5'-GCTGTGCTACGTCGCCCTG-3').

Bio-Rad primers used in the experiments are $\mathrm{CDC} 2$ (CDK1) (qHsaCIP0039501), CCNA2 (qHsaCID0017452), ALDH3A1 (qHsaCID0013758), ESR1 (qHsaCED0042641), ESR2 (qHsaCID0013184) and PGR (qHsaCID0036346).

\section{Live cell imaging}

Cells were seeded at low density on glass bottom plates (MatTek Corporation) coated with $1 \mu \mathrm{g} / \mathrm{ml}$ fibronectin (Sigma-Aldrich). Then, cells were placed on an inverted microscope equipped with a $37^{\circ} \mathrm{C}$ humidified chamber with $5 \% \mathrm{CO}_{2}$, and visualized using a fluorescence microscope (True MultiColor Laser TIRF Leica AM TIRF MC; Leica) equipped with a $63 \times$ oil immersion objective lens (HCX Plan-Apochromat $63 \times / 1.47$ oil CORR TIRF) and an EMCCD camera (C9100-02; Hamamatsu Photonics). Images were acquired with LAS AF6000 modular system software (Leica) at 10-min intervals for a period of 4-5 days.

\section{Mammosphere assay}

Single cells were plated at a density of 1000 per well in ultralow attachment plates (35 mm; Corning). Cells were grown in serum-free DMEM/F12 medium, supplemented with B27 (1:50, Invitrogen), $20 \mathrm{ng} / \mathrm{mL}$ EGF and $20 \mathrm{ng} / \mathrm{mL}$ bFGF (BD Biosciences), and $4 \mu \mathrm{g} / \mathrm{mL}$ heparin (Sigma). Cell mammospheres were cultured for 7-10 days. Then, the mammospheres with diameter $>50 \mu \mathrm{m}$ were counted.

\section{Transwell migration and invasion assays}

Transwell migration assays were performed using Transwell $^{\circledR}$ chamber inserts (Costar, Cambridge, MA) with a porous polycarbonate membrane $(8 \mu \mathrm{M}$ pore size; Corning Costar Incorporated, NY, USA). Briefly, the lower side of the filter was coated with $10 \mu \mathrm{g} / \mathrm{ml}$ fibronectin and blocked with $1 \%$ BSA. For invasion assay, the upper side of the filter was coated with Matrige ${ }^{\circledR}$ according to manufacturer protocol. Approximately $5 \times 10^{4}$ cells were added in the upper chamber, and allowed to migrate through the filter toward the lower chamber containing the indicated factors. In parallel, the same volume of cell suspension was seeded in cell culture multiwell dishes to check for equal cell loading. After 12 or $24 \mathrm{~h}$, non-migrated cells on the upper side of the filter were removed by a cotton swab, followed by fixing with $11 \%$ glutaraldehyde and staining with crystal violet. Microscopic images were then quantified either by cell counting or by converting to a binary image and quantifying the integrated pixel values using ImageJ (NIH). Experiments were repeated at least three times in replicates, showing consistent results.

\section{Data collection and analysis of the online datasets from the breast cancer patient cohorts and cell lines}

The Gene Expression across Normal and Tumor Tissue (GENT) web-accessible database (http://medical-genome. kribb.re.kr/GENT) was used to evaluate Sema4C and PlexinB2 expression patterns in breast cancer tissues. For Sema4C expression, comparing paired tumor and normal tissue was generated by comparing gene expression patterns of 43 breast tumors and their paired normal control from online available dataset GSE15852. Sema4C expression in breast cancer cell lines and non-cancerous cell lines derived from online analysis from http://merav.wi.mit.edu. For meta-analysis of the correlation between Sema4C and PlexinB2 expression and overall survival (OS) or distant metastasis-free survival of breast cancer patients, we used Kaplan-Meier Plotter Platform (http:// kmplot.com/analysis); cBio-portal (http://www.cbioportal.org) was used as for analysis of TCGA breast cancer data [51].

\section{Statistics}

Results are means of at least three different independent experiments \pm standard deviation. Comparisons were made using the two-tailed Student's $t$-test, indicated in graphs as follows: $* * * p<0.001, * * p<0.01, * p \leq 0.05$.

Acknowledgements We gratefully acknowledge the help of S. Giordano, E. Medico, S. Corso, C Isella, M. Rehman, S. Rizzolio, G. Cagnoni and C. Battistini for useful discussion and suggestions. We thank S. Bolla, L. Palmas and F. Sassi for skillful technical assistance; M. Accardo and L. Tarditi for support with experiments in mice; B. Lupo for help with QPCR analysis. The work was supported by grants from the Italian Association for Cancer Research (AIRC-IG \# 201415179 to LT; and AIRC-IG \#15180 to LL), and the Fondazione Piemontese per la Ricerca sul Cancro (grants FPRC-5perMille-MIUR2013 to LT, FPRC-5perMille-Ministero Salute-2013 to LT and FPRC 5xmille Ministero Salute 2015 to LL). 


\section{Compliance with ethical standards}

Conflict of interest The authors declare that they have no conflict of interest.

\section{References}

1. Neufeld G, Kessler O. The semaphorins: versatile regulators of tumour progression and tumour angiogenesis. Nat Rev Cancer. 2008;8:632-45.

2. Rehman M, Tamagnone L. Semaphorins in cancer: biological mechanisms and therapeutic approaches. Semin Cell Dev Biol. 2013;24:179-89.

3. Hota PK, Buck M. Plexin structures are coming: opportunities for multilevel investigations of semaphorin guidance receptors, their cell signaling mechanisms, and functions. Cell Mol Life Sci. 2012;69:3765-805.

4. Gurrapu S, Tamagnone L. Transmembrane semaphorins: multimodal signaling cues in development and cancer. Cell Adhes Migr. 2016;10:675-691.

5. Tamagnone L. Emerging role of semaphorins as major regulatory signals and potential therapeutic targets in cancer. Cancer Cell. 2012;22:145-52.

6. Worzfeld T, Offermanns S. Semaphorins and plexins as therapeutic targets. Nat Rev Drug Discov. 2014;13:603-21.

7. Ye SM, Han M, Kan CY, Yang LL, Yang J, Ma QF, et al. [Expression and clinical significance of Sema4C in esophageal cancer, gastric cancer and rectal cancer]. Zhonghua Yi Xue Za Zhi. 2012;92:1954-8.

8. Li J, Wang Q, Wen R, Liang J, Zhong X, Yang W, et al. MiR-138 inhibits cell proliferation and reverses epithelial-mesenchymal transition in non-small cell lung cancer cells by targeting GIT1 and SEMA4C. J Cell Mol Med. 2015;19:2793-805.

9. Yang Q, Wang Y, Lu X, Zhao Z, Zhu L, Chen S, et al. MiR-125b regulates epithelial-mesenchymal transition via targeting Sema4C in paclitaxel-resistant breast cancer cells. Oncotarget. 2015;6:3268-79.

10. Song J, Li Y. miR-25-3p reverses epithelial-mesenchymal transition via targeting Sema4C in cisplatin-resistance cervical cancer cells. Cancer Sci. 2016;108:23-31.

11. Gao Q, Wei JC, Yang J, Liu D, Wu MF, Qiao L, et al. Tumorassociated lymphatic endothelial cells promote lymphatic metastasis by highly expressing and secreting SEMA4C. Clin Cancer Res. 2016;23:214-24.

12. Deng S, Hirschberg A, Worzfeld T, Penachioni JY, Korostylev A, Swiercz JM, et al. Plexin-B2, but not Plexin-B1, critically modulates neuronal migration and patterning of the developing nervous system in vivo. The. J Neurosci. 2007;27:6333-47.

13. Witherden Deborah A, Watanabe M, Garijo O, Rieder Stephanie E, Sarkisyan G, Cronin Shane JF, et al. The CD100 receptor interacts with its PlexinB2 ligand to regulate epidermal $\gamma \delta \mathrm{T}$ cell function. Immunity. 2012;37:314-25.

14. Azzarelli R, Pacary E, Garg R, Garcez P, van den Berg D, Riou P, et al. An antagonistic interaction between PlexinB2 and Rnd3 controls RhoA activity and cortical neuron migration. Nat Commun. 2014;5:3405

15. Perälä N, Jakobson M, Ola R, Fazzari P, Penachioni JY, Nymark $\mathrm{M}$, et al. Sema4C-Plexin B2 signalling modulates ureteric branching in developing kidney. Differentiation. 2011;81:81-91.

16. Xia J, Swiercz JM, Banon-Rodriguez I, Matkovic I, Federico G, Sun T, et al. Semaphorin-plexin signaling controls mitotic spindle orientation during epithelial morphogenesis and repair. Dev Cell. 2015;33:299-313.

17. Aurandt J, Vikis HG, Gutkind JS, Ahn N, Guan KL. The semaphorin receptor plexin-B1 signals through a direct interaction with the Rho-specific nucleotide exchange factor, LARG. Proc Natl Acad Sci USA. 2002;99:12085-90.

18. Perrot V, Vazquez-Prado J, Gutkind JS. Plexin B regulates Rho through the guanine nucleotide exchange factors leukemiaassociated Rho GEF (LARG) and PDZ-RhoGEF. J Biol Chem. 2002;277:43115-20.

19. Swiercz JM, Kuner R, Behrens J, Offermanns S. Plexin-B1 directly interacts with PDZ-RhoGEF/LARG to regulate RhoA and growth cone morphology. Neuron. 2002;35:51-63.

20. Fritz G, Brachetti C, Bahlmann F, Schmidt M, Kaina B. Rho GTPases in human breast tumours: expression and mutation analyses and correlation with clinical parameters. Br J Cancer. 2002;87:635-44.

21. Shang X, Marchioni F, Sipes N, Evelyn CR, Jerabek-Willemsen $\mathrm{M}$, Duhr S, et al. Rational design of small molecule inhibitors targeting RhoA subfamily Rho GTPases. Chem Biol. 2012;19:699-710.

22. Pille JY, Denoyelle C, Varet J, Bertrand JR, Soria J, Opolon P, et al. Anti-RhoA and anti-RhoC siRNAs inhibit the proliferation and invasiveness of MDA-MB-231 breast cancer cells in vitro and in vivo. Mol Ther. 2005;11:267-74.

23. Chan $\mathrm{CH}$, Lee SW, Li CF, Wang J, Yang WL, Wu CY, et al. Deciphering the transcriptional complex critical for RhoA gene expression and cancer metastasis. Nat Cell Biol. 2010;12:457-67.

24. Zhang C, Wang HJ, Bao QC, Wang L, Guo TK, Chen WL, et al. NRF2 promotes breast cancer cell proliferation and metastasis by increasing RhoA/ROCK pathway signal transduction. Oncotarget. 2016;7:73593-606.

25. Hauser AD, Bergom C, Schuld NJ, Chen X, Lorimer EL, Huang J, et al. The SmgGDS splice variant SmgGDS-558 is a key promoter of tumor growth and RhoA signaling in breast cancer. Mol Cancer Res. 2014;12:130-42.

26. Patel RA, Forinash KD, Pireddu R, Sun Y, Sun N, Martin MP, et al. RKI-1447 is a potent inhibitor of the Rho-associated ROCK kinases with anti-invasive and antitumor activities in breast cancer. Cancer Res. 2012;72:5025-34.

27. Naderi A, Teschendorff AE, Barbosa-Morais NL, Pinder SE, Green AR, Powe DG, et al. A gene-expression signature to predict survival in breast cancer across independent data sets. Oncogene. 2007;26:1507-16.

28. Holliday DL, Speirs V. Choosing the right cell line for breast cancer research. Breast Cancer Res. 2011;13:215.

29. Kümper S, Mardakheh FK, McCarthy A, Yeo M, Stamp GW, Paul A, et al. Rho-associated kinase (ROCK) function is essential for cell cycle progression, senescence and tumorigenesis. eLife. 2016;5:e12994.

30. Krusche B, Ottone C, Clements MP, Johnstone ER, Goetsch K, Lieven $\mathrm{H}$, et al. EphrinB2 drives perivascular invasion and proliferation of glioblastoma stem-like cells. eLife. 2016;5:e14845.

31. McColl B, Garg R, Riou P, Riento K, Ridley AJ. Rnd3-induced cell rounding requires interaction with Plexin-B2. J Cell Sci. 2016;129:4046-56.

32. Paldy E, Simonetti M, Worzfeld T, Bali KK, Vicuna L, Offermanns $\mathrm{S}$, et al. Semaphorin 4C Plexin-B2 signaling in peripheral sensory neurons is pronociceptive in a model of inflammatory pain. Nat Commun. 2017;8:176.

33. Worzfeld T, Swiercz JM, Senturk A, Genz B, Korostylev A, Deng $\mathrm{S}$, et al. Genetic dissection of plexin signaling in vivo. Proc Natl Acad Sci USA. 2014;111:2194-9.

34. Casteel DE, Turner S, Schwappacher R, Rangaswami H, Su-Yuo $\mathrm{J}$, Zhuang $\mathrm{S}$, et al. Rho isoform-specific interaction with IQGAP1 promotes breast cancer cell proliferation and migration. $\mathrm{J}$ Biol Chem. 2012;287:38367-78.

35. Wang JB, Erickson JW, Fuji R, Ramachandran S, Gao P, Dinavahi $R$, et al. Targeting mitochondrial glutaminase activity inhibits oncogenic transformation. Cancer Cell. 2010;18:207-19. 
36. Swiercz JM, Kuner R, Offermanns S. Plexin-B1/RhoGEF-mediated RhoA activation involves the receptor tyrosine kinase ErbB2. J Cell Biol. 2004;165:869-80.

37. Swiercz JM, Worzfeld T, Offermanns S. ErbB-2 and met reciprocally regulate cellular signaling via plexin-B1. J Biol Chem. 2008;283:1893-901.

38. Aranda V, Haire T, Nolan ME, Calarco JP, Rosenberg AZ, Fawcett JP, et al. Par6-aPKC uncouples ErbB2 induced disruption of polarized epithelial organization from proliferation control. Nat Cell Biol. 2006;8:1235-45.

39. Nakayama M, Goto TM, Sugimoto M, Nishimura T, Shinagawa T, Ohno S, et al. Rho-kinase phosphorylates PAR-3 and disrupts PAR complex formation. Dev Cell. 2008;14:205-15.

40. Shibue T, Weinberg RA. EMT, CSCs, and drug resistance: the mechanistic link and clinical implications. Nat Rev Clin Oncol. 2017;14:611-29 (advance online publication).

41. Ye X, Weinberg RA. Epithelial-mesenchymal plasticity: a central regulator of cancer progression. Trends Cell Biol. 2015;25:675-86.

42. Mani SA, Guo W, Liao MJ, Eaton EN, Ayyanan A, Zhou AY, et al. The epithelial-mesenchymal transition generates cells with properties of stem cells. Cell . 2008;133:704-15.

43. Lu MS, Johnston CA. Molecular pathways regulating mitotic spindle orientation in animal cells. Development. 2013;140:1843-56.

44. Wodarz A, Nathke I. Cell polarity in development and cancer. Nat Cell Biol. 2007;9:1016-24.

45. Frisch SM, Schaller M, Cieply B. Mechanisms that link the oncogenic epithelial-mesenchymal transition to suppression of anoikis. J Cell Sci. 2013;126(Pt 1):21-9.

46. Lee JY, Won HY, Park JH, Kim HY, Choi HJ, Shin DH, et al. MEL-18 loss mediates estrogen receptor-alpha downregulation and hormone independence. J Clin Invest. 2015;125:1801-14.

47. Liang Y-K, Zeng D, Xiao Y-S, Wu Y, Ouyang Y-X, Chen M, et al. MCAM/CD146 promotes tamoxifen resistance in breast cancer cells through induction of epithelial-mesenchymal transition, decreased ER $\alpha$ expression and AKT activation. Cancer Lett. 2017;386:65-76.

48. Pancholi S, Lykkesfeldt AE, Hilmi C, Banerjee S, Leary A, Drury $\mathrm{S}$, et al. ERBB2 influences the subcellular localization of the estrogen receptor in tamoxifen-resistant MCF-7 cells leading to the activation of AKT and RPS6KA2. Endocr Relat Cancer. 2008;15:985-1002.

49. Lin CY, Strom A, Vega VB, Kong SL, Yeo AL, Thomsen JS, et al. Discovery of estrogen receptor alpha target genes and response elements in breast tumor cells. Genome Biol. 2004;5: R66.

50. Piva M, Domenici G, Iriondo O, Rabano M, Simoes BM, Comaills V, et al. Sox2 promotes tamoxifen resistance in breast cancer cells. EMBO Mol Med. 2014;6:66-79.

51. Ciriello G, Gatza ML, Beck AH, Wilkerson MD, Rhie SK, Pastore A, et al. Comprehensive molecular portraits of invasive lobular breast cancer. Cell. 2015;163:506-19.

52. Osborne CK, Hobbs K, Clark GM. Effect of estrogens and antiestrogens on growth of human breast cancer cells in athymic nude mice. Cancer Res. 1985;45:584-90.

53. Levenson AS, Jordan VC. MCF-7: the first hormone-responsive breast cancer cell line. Cancer Res. 1997;57:3071-8.

54. Friedel RH, Kerjan G, Rayburn H, Schuller U, Sotelo C, TessierLavigne $\mathrm{M}$, et al. Plexin-B2 controls the development of cerebellar granule cells. J Neurosci. 2007;27:3921-32.

55. Maier V, Jolicoeur C, Rayburn H, Takegahara N, Kumanogoh A, Kikutani H, et al. Semaphorin $4 \mathrm{C}$ and $4 \mathrm{G}$ are ligands of Plexin-B2 required in cerebellar development. Mol Cell Neurosci. 2011;46:419-31.

56. Dull T, Zufferey R, Kelly M, Mandel RJ, Nguyen M, Trono D, et al. A third-generation lentivirus vector with a conditional packaging system. J Virol. 1998;72:8463-71.

57. Toyoshima F, Nishida E. Integrin-mediated adhesion orients the spindle parallel to the substratum in an EB1- and myosin Xdependent manner. EMBO J. 2007;26:1487-98. 